\title{
Explicit monotone iterative sequences for positive solutions of a fractional differential system with coupled integral boundary conditions on a half-line
}

\author{
Haiyan Zhang ${ }^{1}$, Yongqing Wang ${ }^{2 *}$ (D) and Jiafa $\mathrm{Xu}^{3}$
}

\section{"Correspondence: \\ wyqing9801@163.com \\ ${ }^{2}$ School of Mathematical Sciences, \\ Qufu Normal University, Qufu \\ 273165, Shandong, P.R. China \\ Full list of author information is \\ available at the end of the article}

\begin{abstract}
In this paper we consider a fractional differential system with coupled integral boundary value problems on a half-line, where the nonlinearity terms depend on unknown functions and the lower-order fractional derivative of unknown functions, and the fractional infinite boundary value conditions depend on the coupled infinite integral of unknown functions. By virtue of the monotone iterative technique, we find two explicit monotone iterative sequences which converge to the positive minimal and maximal solutions when the nonlinearities can satisfy certain nonlinear growth conditions.
\end{abstract}

Keywords: Monotone iterative technique; Iterative sequences; Fractional differential system; Integral boundary value problems; Half-line

\section{Springer}

\section{Introduction}

Fractional-order differential equations is a natural generalization of the case of integer order, which has become the focus of attention involving various kinds of boundary conditions because of the wide application in mathematical models and applied sciences. Some latest results on the topic can be found in a series of papers [1-15] and the references therein. In particular, a monotone iterative technique is believed to be an efficient and important method to deal with sequences of monotone solutions for initial and boundary value problems. For some applications of this method to nonlinear fractional differential equations, see [16-24]. We also note that there are some results about monotone iterative solution of a single fractional order equation on a half-line, see [25-29].

In [25] Zhang considered a nonlinear fractional boundary value problem on a half-line

$$
\left\{\begin{array}{l}
D^{\alpha} u(t)+f\left(t, u(t), D^{\alpha-1} u(t)\right)=0, \quad t \in(0,+\infty), \alpha \in(1,2], \\
u(0)=0, \quad D^{\alpha-1} u(+\infty)=\beta u(\xi), \quad \beta>0,
\end{array}\right.
$$

(c) The Author(s) 2020. This article is licensed under a Creative Commons Attribution 4.0 International License, which permits use, sharing, adaptation, distribution and reproduction in any medium or format, as long as you give appropriate credit to the original author(s) and the source, provide a link to the Creative Commons licence, and indicate if changes were made. The images or other third party material in this article are included in the article's Creative Commons licence, unless indicated otherwise in a credit line to the material. If material is not included in the article's Creative Commons licence and your intended use is not permitted by statutory regulation or exceeds the permitted use, you will need to obtain permission directly from the copyright holder. To view a copy of this licence, visit http://creativecommons.org/licenses/by/4.0/. 
where $f \in C(J \times \mathbb{R} \times \mathbb{R}, \mathbb{R})$. By utilizing the monotone iterative technique, iterative sequences of the positive extremal solutions were acquired.

In [27] Pei et al. studied the Hadamard fractional integro-differential equation on an infinite interval

$$
\left\{\begin{array}{l}
D^{\alpha} u(t)+f\left(t, u(t), I^{r} u(t), D^{\alpha-1} u(t)\right)=0, \quad 1<\alpha<2, t \in(1,+\infty), \\
u(1)=0, \quad D^{\alpha-1} u(\infty)=\sum_{i=1}^{m} \lambda_{i} I^{\beta_{i}} u(\eta)
\end{array}\right.
$$

where $I^{(\cdot)}$ is the Hadamard fractional integral and $\alpha, \eta, \beta_{i}, \lambda_{i}(i=1,2, \ldots, m)$ are some given constants with $\Gamma(\alpha)>\sum_{i=1}^{m} \frac{\lambda_{i} \Gamma(\alpha)}{\Gamma\left(\alpha+\beta_{i}\right)}(\log \eta)^{\alpha+\beta_{i}-1}$. By using the monotone iterative technique, the existence of positive solutions was established.

On the other hand, integral boundary conditions are considered to be more reasonable than the local boundary conditions, which can depict phenomena of heat transmission, population dynamics, blood flow, etc. A large number of results about fractional differential equations with integral boundary condition have been obtained, see $[9,10,30-43]$ and the references cited therein. Meanwhile, we note that the coupled systems of fractionalorder differential equations have also attracted much attention due to their extensive applications, we refer to $[3,9,10,14,22,29,32-43]$.

In [9] Jiang et al. utilized the fixed point index to construct the existence of positive solutions for the following system on a finite interval:

$$
\left\{\begin{array}{l}
D^{\beta} u(t)+f_{1}(t, u(t), v(t))=0, \quad 1<t<e, \\
D^{\beta} v(t)+f_{2}(t, u(t), v(t))=0, \quad 1<t<e, \\
u(1)=v(1)=u^{\prime}(1)=v^{\prime}(1)=0, \\
u(e)=\int_{1}^{e} h(s) v(s) \frac{d s}{s} \\
v(e)=\int_{1}^{e} g(s) u(s) \frac{d s}{s}
\end{array}\right.
$$

where the nonlinearities $f_{i}(i=1,2)$ can grow superlinearly and sublinearly, and boundary value conditions depend on the coupled integral of unknown functions.

In [29] a coupled system of fractional differential equations on an infinite interval is studied

$$
\left\{\begin{array}{l}
D^{\alpha} u(t)+\varphi\left(t, v(t), D^{\gamma_{1}} v(t)\right)=0, \quad \alpha \in(2,3], \gamma_{1} \in(0,1), \\
D^{\beta} v(t)+\psi\left(t, u(t), D^{\gamma_{2}} u(t)\right)=0, \quad \beta \in(2,3], \gamma_{2} \in(0,1), \\
I^{3-\alpha} u(0)=0, \quad D^{\alpha-2} u(0)=\int_{0}^{h} g_{1}(s) u(s) \mathrm{d} s, \quad D^{\alpha-1} u(+\infty)=M u(\xi)+a, \\
I^{3-\beta} v(0)=0, \quad D^{\beta-2} v(0)=\int_{0}^{h} g_{2}(s) v(s) \mathrm{d} s, \quad D^{\beta-1} v(+\infty)=N v(\eta)+b,
\end{array}\right.
$$

where $t \in J=[0,+\infty), \varphi, \psi \in C(J \times \mathbb{R} \times \mathbb{R}, J), M, N$ are real numbers satisfying $0<M \xi^{\alpha-1}<$ $\Gamma(\alpha), 0<N \eta^{\beta-1}<\Gamma(\beta), \xi, \eta, h>0$, and $a, b \in \mathbb{R}^{+}, g_{1}, g_{2} \in L^{1}[0, h]$ are nonnegative functions, the nonlinear terms $\varphi, \psi$ and boundary conditions of the system are not coupled. 
In [32] Aljoudi et al. studied the sequential fractional differential equations on a finite interval

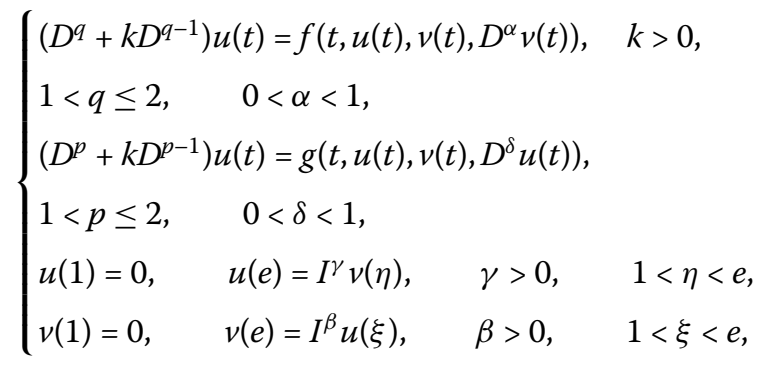

where $D^{(\cdot)}$ and $I^{(\cdot)}$ denote the Hadamard fractional derivative and Hadamard fractional integral, $f, g:[1, e] \times \mathbb{R}^{3} \rightarrow \mathbb{R}$ are given continuous functions, and boundary value conditions depend on the coupled fractional integral of unknown functions.

Inspired by the works above, in this paper we utilize the monotone iterative technique to study the existence of positive extremal solutions of a fractional differential system on a half-line

$$
\left\{\begin{array}{l}
D^{\alpha} u(t)+\varphi\left(t, u(t), v(t), D^{\beta-1} v(t)\right)=0, \quad 2<\alpha \leq 3, \\
D^{\beta} v(t)+\psi\left(t, u(t), v(t), D^{\alpha-1} u(t)\right)=0, \quad 2<\beta \leq 3, \\
u(0)=u^{\prime}(0)=0, \quad D^{\alpha-1} u(+\infty)=\int_{0}^{+\infty} h(t) v(t) \mathrm{d} t, \\
v(0)=v^{\prime}(0)=0, \quad D^{\beta-1} v(+\infty)=\int_{0}^{+\infty} g(t) u(t) \mathrm{d} t,
\end{array}\right.
$$

where $D^{\alpha}, D^{\beta}$ are the Riemann-Liouville fractional derivatives. Here we emphasize that the nonlinearity terms $\varphi$, $\psi$ include not only unknown functions, but also the lower-order fractional derivative of unknown functions. By the way, the fractional infinite boundary value conditions depend on the coupled infinite integral of unknown functions. To the best of the authors' knowledge, the system with coupled infinite integral boundary value conditions is yet to be investigated. $\varphi, \psi$ satisfy the following assumptions:

(C0) $\varphi, \psi \in C(J \times \mathbb{R} \times \mathbb{R} \times \mathbb{R}, J), J=[0,+\infty)$.

(C1) $h(t), g(t) \in L[0,+\infty)$ with $\int_{0}^{+\infty} h(t) t^{\beta-1} \mathrm{~d} t=\Upsilon_{1}, \int_{0}^{+\infty} g(t) t^{\alpha-1} \mathrm{~d} t=\Upsilon_{2}, \Upsilon_{1} \Upsilon_{2}<$ $\Gamma(\alpha) \Gamma(\beta)$.

(C2) The nonnegative functions $a_{i}(t), b_{i}(t) \in L[0,+\infty)(i=0,1,2,3)$ and constants $0 \leq$ $\lambda_{k}, \tau_{k}<1(k=1,2,3)$ satisfy

$$
|\varphi(t, u, v, w)| \leq a_{0}(t)+a_{1}(t)|u|^{\lambda_{1}}+a_{2}(t)|v|^{\lambda_{2}}+a_{3}(t)|w|^{\lambda_{3}}, \quad u, v, w \in \mathbb{R}, \forall t \in J,
$$

with

$$
\begin{aligned}
& \int_{0}^{+\infty} a_{0}(t) \mathrm{d} t=a_{0}^{*}<+\infty, \quad \int_{0}^{+\infty} a_{1}(t)\left(1+t^{\alpha+\beta-1}\right)^{\lambda_{1}} \mathrm{~d} t=a_{1}^{*}<+\infty, \\
& \int_{0}^{+\infty} a_{2}(t)\left(1+t^{\alpha+\beta-1}\right)^{\lambda_{2}} \mathrm{~d} t=a_{2}^{*}<+\infty, \quad \int_{0}^{+\infty} a_{3}(t) \mathrm{d} t=a_{3}^{*}<+\infty,
\end{aligned}
$$

and

$$
|\psi(t, u, v, z)| \leq b_{0}(t)+b_{1}(t)|u|^{\tau_{1}}+b_{2}(t)|v|^{\tau_{2}}+a_{3}(t)|z|^{\tau_{3}}, \quad u, v, z \in \mathbb{R}, \forall t \in J,
$$


with

$$
\begin{aligned}
& \int_{0}^{+\infty} b_{0}(t) \mathrm{d} t=b_{0}^{*}<+\infty, \quad \int_{0}^{+\infty} b_{1}(t)\left(1+t^{\alpha+\beta-1}\right)^{\tau_{1}} \mathrm{~d} t=b_{1}^{*}<+\infty, \\
& \int_{0}^{+\infty} b_{2}(t)\left(1+t^{\alpha+\beta-1}\right)^{\tau_{2}} \mathrm{~d} t=b_{2}^{*}<+\infty, \quad \int_{0}^{+\infty} b_{3}(t) \mathrm{d} t=b_{3}^{*}<+\infty .
\end{aligned}
$$

(C3) $\varphi(t, u, v, w)$ and $\psi(t, u, v, z)$ are increasing with respect to the variables $u, v, w$ and $u, v, z$, and $\varphi(t, 0,0,0) \not \equiv 0, \psi(t, 0,0,0) \not \equiv 0, \forall t \in J$.

For convenience, we set $\Delta=\Gamma(\alpha) \Gamma(\beta)-\Upsilon_{1} \Upsilon_{2}, \Theta=\max \left\{\Gamma(\beta)+\Upsilon_{1}, \Gamma(\alpha)+\Upsilon_{2}\right.$, $\left.\Gamma(\alpha)\left(\Gamma(\beta)+\Upsilon_{1}\right), \Gamma(\beta)\left(\Gamma(\alpha)+\Upsilon_{2}\right)\right\}, \Lambda=\frac{\Theta}{\Delta}$.

\section{Preliminaries}

In this section we only list some definitions and lemmas of the Riemann-Liouville fractional integral and derivative; for more details, we refer the readers to [1].

Definition 2.1 (see [1]) The Riemann-Liouville fractional integral of order $q>0$ for an integrable function $g$ is defined as

$$
I^{q} g(x)=\frac{1}{\Gamma(q)} \int_{0}^{x}(x-t)^{q-1} g(t) \mathrm{d} t
$$

provided that the integral exists.

Definition 2.2 (see [1]) The Riemann-Liouville fractional derivative of order $q>0$ for an integrable function $g$ is defined as

$$
D^{q} g(x)=\frac{1}{\Gamma(n-q)}\left(\frac{d}{d x}\right)^{n} \int_{0}^{x}(x-t)^{n-q-1} g(t) \mathrm{d} t,
$$

where $n=[q]+1,[q]$ is the smallest integer greater than or equal to $q$, provided that the right-hand side is pointwise defined on $(0,+\infty)$.

Lemma 2.3 (see [1]) Let $q>0$ and $u \in C(0,+\infty) \cap L(0,+\infty)$. Then the general solution of fractional differential equation $D^{q} u(t)=0$ is

$$
u(t)=c_{1} t^{q-1}+c_{2} t^{q-2}+\cdots+c_{n} t^{q-n},
$$

where $c_{i} \in \mathbb{R}, i=1,2, \ldots, n$, and $n-1<q<n$.

Lemma 2.4 Let $x, y \in C(0,+\infty) \cap L(0,+\infty)$ and assumption (C1) be satisfied. Then the fractional differential system with coupled integral boundary conditions

$$
\left\{\begin{array}{l}
D^{\alpha} u(t)+x(t)=0, \quad 2<\alpha \leq 3, \\
D^{\beta} v(t)+y(t)=0, \quad 2<\beta \leq 3, \\
u(0)=u^{\prime}(0)=0, \quad D^{\alpha-1} u(+\infty)=\int_{0}^{+\infty} h(t) v(t) \mathrm{d} t, \\
v(0)=v^{\prime}(0)=0, \quad D^{\beta-1} v(+\infty)=\int_{0}^{+\infty} g(t) u(t) \mathrm{d} t,
\end{array}\right.
$$


has a solution which can take the integral representation

$$
\left\{\begin{array}{l}
u(t)=\int_{0}^{+\infty} K_{1}(t, s) x(s) \mathrm{d} s+\int_{0}^{+\infty} K_{3}(t, s) y(s) \mathrm{d} s, \\
v(t)=\int_{0}^{+\infty} K_{2}(t, s) y(s) \mathrm{d} s+\int_{0}^{+\infty} K_{4}(t, s) x(s) \mathrm{d} s
\end{array}\right.
$$

where

$$
\begin{aligned}
& K_{1}(t, s)=K_{11}(t, s)+K_{12}(t, s), \quad K_{2}(t, s)=K_{21}(t, s)+K_{22}(t, s) \\
& K_{3}(t, s)=\frac{\Gamma(\beta) t^{\alpha-1}}{\Delta} \int_{0}^{+\infty} h(t) K_{21}(t, s) \mathrm{d} t \\
& K_{4}(t, s)=\frac{\Gamma(\alpha) t^{\beta-1}}{\Delta} \int_{0}^{+\infty} g(t) K_{11}(t, s) \mathrm{d} t
\end{aligned}
$$

with

$$
\begin{aligned}
& K_{11}(t, s)=\frac{1}{\Gamma(\alpha)} \begin{cases}t^{\alpha-1}-(t-s)^{\alpha-1}, & 0 \leq s \leq t \leq+\infty, \\
t^{\alpha-1}, & 0 \leq t \leq s \leq+\infty,\end{cases} \\
& K_{12}(t, s)=\frac{\Upsilon_{1} t^{\alpha-1}}{\Delta} \int_{0}^{+\infty} g(t) K_{11}(t, s) \mathrm{d} t, \\
& K_{21}(t, s)=\frac{1}{\Gamma(\beta)} \begin{cases}t^{\beta-1}-(t-s)^{\beta-1}, & 0 \leq s \leq t \leq+\infty, \\
t^{\beta-1}, & 0 \leq t \leq s \leq+\infty,\end{cases} \\
& K_{22}(t, s)=\frac{\Upsilon_{2} t^{\beta-1}}{\Delta} \int_{0}^{+\infty} h(t) K_{21}(t, s) \mathrm{d} t .
\end{aligned}
$$

Proof By Lemma 2.3, we can turn system (2.1) into an equivalent integral system

$$
\left\{\begin{array}{l}
u(t)=-\frac{1}{\Gamma(\alpha)} \int_{0}^{t}(t-s)^{\alpha} x(s) \mathrm{d} s+c_{1} t^{\alpha-1}+c_{2} t^{\alpha-2}+c_{3} t^{\alpha-3}, \\
v(t)=-\frac{1}{\Gamma(\beta)} \int_{0}^{t}(t-s)^{\beta} y(s) \mathrm{d} s+d_{1} t^{\beta-1}+d_{2} t^{\beta-2}+d_{3} t^{\beta-3},
\end{array}\right.
$$

where $c_{i}, d_{i} \in \mathbb{R}(i=1,2,3)$. Notice that $u(0)=u^{\prime}(0)=0$ and $v(0)=v^{\prime}(0)=0$, we have $c_{2}=$ $c_{3}=d_{2}=d_{3}=0$. From (2.4) we have

$$
\left\{\begin{array}{l}
u(t)=-\frac{1}{\Gamma(\alpha)} \int_{0}^{t}(t-s)^{\alpha} x(s) \mathrm{d} s+c_{1} t^{\alpha-1}, \\
v(t)=-\frac{1}{\Gamma(\beta)} \int_{0}^{t}(t-s)^{\beta} y(s) \mathrm{d} s+d_{1} t^{\beta-1} .
\end{array}\right.
$$

As a result,

$$
\left\{\begin{array}{l}
D^{\alpha-1} u(t)=c_{1} \Gamma(\alpha)-\int_{0}^{t} x(s) \mathrm{d} s \\
D^{\beta-1} v(t)=d_{1} \Gamma(\beta)-\int_{0}^{t} y(s) \mathrm{d} s .
\end{array}\right.
$$

That is,

$$
\left\{\begin{array}{l}
D^{\alpha-1} u(+\infty)=c_{1} \Gamma(\alpha)-\int_{0}^{+\infty} x(s) \mathrm{d} s, \\
D^{\beta-1} v(+\infty)=d_{1} \Gamma(\beta)-\int_{0}^{+\infty} y(s) \mathrm{d} s .
\end{array}\right.
$$


By means of conditions $D^{\alpha-1} u(+\infty)=\int_{0}^{+\infty} h(t) v(t) \mathrm{d} t$ and $D^{\beta-1} v(+\infty)=\int_{0}^{+\infty} g(t) u(t) \mathrm{d} t$, we have

$$
\left\{\begin{array}{l}
c_{1}=\frac{1}{\Gamma(\alpha)} \int_{0}^{+\infty} h(t) v(t) \mathrm{d} t+\frac{1}{\Gamma(\alpha)} \int_{0}^{+\infty} x(s) \mathrm{d} s \\
d_{1}=\frac{1}{\Gamma(\beta)} \int_{0}^{+\infty} g(t) u(t) \mathrm{d} t+\frac{1}{\Gamma(\beta)} \int_{0}^{+\infty} y(s) \mathrm{d} s
\end{array}\right.
$$

Submitting (2.8) to (2.5), we obtain

$$
\left\{\begin{aligned}
u(t) & =-\frac{1}{\Gamma(\alpha)} \int_{0}^{t}(t-s)^{\alpha} x(s) \mathrm{d} s+\frac{t^{\alpha-1}}{\Gamma(\alpha)}\left[\int_{0}^{+\infty} h(t) v(t) \mathrm{d} t+\int_{0}^{+\infty} x(s) \mathrm{d} s\right] \\
& =\int_{0}^{\infty} K_{11}(t, s) x(s) \mathrm{d} s+\frac{t^{\alpha-1}}{\Gamma(\alpha)} \int_{0}^{+\infty} h(t) v(t) \mathrm{d} t, \\
v(t) & =-\frac{1}{\Gamma(\beta)} \int_{0}^{t}(t-s)^{\beta} y(s) \mathrm{d} s+\frac{t^{\beta-1}}{\Gamma(\beta)}\left[\int_{0}^{+\infty} g(t) u(t) \mathrm{d} t+\int_{0}^{+\infty} y(s) \mathrm{d} s\right] \\
& =\int_{0}^{\infty} K_{21}(t, s) y(s) \mathrm{d} s+\frac{t^{\beta-1}}{\Gamma(\beta)} \int_{0}^{+\infty} g(t) u(t) \mathrm{d} t .
\end{aligned}\right.
$$

Multiplying both sides of equality (2.9) by $g(t)$ and $h(t)$ and integrating from 0 to $+\infty$, we have

$$
\left\{\begin{array}{l}
\int_{0}^{+\infty} g(t) u(t) \mathrm{d} t=\int_{0}^{+\infty} g(t) \int_{0}^{+\infty} K_{11}(t, s) x(s) \mathrm{d} s \mathrm{~d} t+\frac{\Upsilon_{2}}{\Gamma(\alpha)} \int_{0}^{+\infty} h(t) v(t) \mathrm{d} t \\
\int_{0}^{+\infty} h(t) v(t) \mathrm{d} t=\int_{0}^{+\infty} h(t) \int_{0}^{+\infty} K_{21}(t, s) y(s) \mathrm{d} s \mathrm{~d} t+\frac{\Upsilon_{1}}{\Gamma(\beta)} \int_{0}^{+\infty} g(t) u(t) \mathrm{d} t .
\end{array}\right.
$$

Then

$$
\left\{\begin{aligned}
\int_{0}^{+\infty} g(t) u(t) \mathrm{d} t= & \frac{\Gamma(\alpha) \Gamma(\beta)}{\Delta}\left[\int_{0}^{+\infty} g(t) \int_{0}^{+\infty} K_{11}(t, s) x(s) \mathrm{d} s \mathrm{~d} t\right. \\
& \left.+\frac{\Upsilon_{2}}{\Gamma(\alpha)} \int_{0}^{+\infty} h(t) \int_{0}^{+\infty} K_{21}(t, s) y(s) \mathrm{d} s \mathrm{~d} t\right], \\
\int_{0}^{+\infty} h(t) v(t) \mathrm{d} t= & \frac{\Gamma(\alpha) \Gamma(\beta)}{\Delta}\left[\frac{\Upsilon_{1}}{\Gamma(\beta)} \int_{0}^{+\infty} g(t) \int_{0}^{+\infty} K_{11}(t, s) x(s) \mathrm{d} s \mathrm{~d} t\right. \\
& \left.+\int_{0}^{+\infty} h(t) \int_{0}^{+\infty} K_{21}(t, s) y(s) \mathrm{d} s \mathrm{~d} t\right] .
\end{aligned}\right.
$$

Submitting (2.10) to (2.9), we have

$$
\left\{\begin{aligned}
u(t)= & \int_{0}^{+\infty} K_{11}(t, s) x(s) \mathrm{d} s+\frac{t^{\alpha-1} \Upsilon_{1}}{\Delta} \int_{0}^{+\infty} g(t) \int_{0}^{+\infty} K_{11}(t, s) x(s) \mathrm{d} s \mathrm{~d} t \\
& +\frac{t^{\alpha-1} \Gamma(\beta)}{\Delta} \int_{0}^{+\infty} h(t) \int_{0}^{+\infty} K_{21}(t, s) y(s) \mathrm{d} s \mathrm{~d} t \\
= & \int_{0}^{\infty} K_{11}(t, s) x(s) \mathrm{d} s+\int_{0}^{\infty} K_{12}(t, s) x(s) \mathrm{d} s+\int_{0}^{\infty} K_{3}(t, s) y(s) \mathrm{d} s \\
= & \int_{0}^{\infty} K_{1}(t, s) x(s) \mathrm{d} s+\int_{0}^{\infty} K_{3}(t, s) y(s) \mathrm{d} s, \\
v(t)= & \int_{0}^{+\infty} K_{21}(t, s) y(s) \mathrm{d} s+\frac{t^{\beta-1} \Gamma(\alpha)}{\Delta} \int_{0}^{+\infty} g(t) \int_{0}^{+\infty} K_{11}(t, s) x(s) \mathrm{d} s \mathrm{~d} t \\
& +\frac{t^{\beta-1} \Upsilon_{2}}{\Delta} \int_{0}^{+\infty} h(t) \int_{0}^{+\infty} K_{21}(t, s) y(s) \mathrm{d} s \mathrm{~d} t \\
= & \int_{0}^{+\infty} K_{21}(t, s) y(s) \mathrm{d} s+\int_{0}^{+\infty} K_{22}(t, s) y(s) \mathrm{d} s+\int_{0}^{+\infty} K_{4}(t, s) x(s) \mathrm{d} s \\
= & \int_{0}^{+\infty} K_{2}(t, s) y(s) \mathrm{d} s+\int_{0}^{+\infty} K_{4}(t, s) x(s) \mathrm{d} s .
\end{aligned}\right.
$$

The proof is completed.

Lemma 2.5 For $(s, t) \in J \times J$, if assumption $(\mathrm{C} 1)$ is satisfied, then

$$
\begin{array}{ll}
0 \leq K_{1}(t, s) \leq \frac{\Gamma(\beta) t^{\alpha-1}}{\Delta}, & 0 \leq \frac{K_{1}(t, s)}{1+t^{\alpha+\beta-1}} \leq \frac{\Gamma(\beta)}{\Delta} \\
0 \leq K_{2}(t, s) \leq \frac{\Gamma(\alpha) t^{\beta-1}}{\Delta}, & 0 \leq \frac{K_{2}(t, s)}{1+t^{\alpha+\beta-1}} \leq \frac{\Gamma(\alpha)}{\Delta}
\end{array}
$$




$$
\begin{array}{ll}
0 \leq K_{3}(t, s) \leq \frac{\Upsilon_{1} t^{\alpha-1}}{\Delta}, & 0 \leq \frac{K_{3}(t, s)}{1+t^{\alpha+\beta-1}} \leq \frac{\Upsilon_{1}}{\Delta}, \\
0 \leq K_{4}(t, s) \leq \frac{\Upsilon_{2} t^{\beta-1}}{\Delta}, & 0 \leq \frac{K_{4}(t, s)}{1+t^{\alpha+\beta-1}} \leq \frac{\Upsilon_{2}}{\Delta} .
\end{array}
$$

Proof From (2.3), it is obvious that

$$
0 \leq K_{11}(t, s) \leq \frac{t^{\alpha-1}}{\Gamma(\alpha)}, \quad \forall(t, s) \in J \times J
$$

and

$$
0 \leq K_{12}(t, s) \leq \frac{\Upsilon_{1} t^{\alpha-1}}{\Delta} \int_{0}^{+\infty} \frac{g(t) t^{\alpha-1}}{\Gamma(\alpha)} \mathrm{d} t=\frac{\Upsilon_{1} \Upsilon_{2} t^{\alpha-1}}{\Gamma(\alpha) \Delta}, \quad \forall(t, s) \in J \times J .
$$

Thus

$$
0 \leq K_{1}(t, s)=K_{11}(t, s)+K_{12}(t, s) \leq \frac{\Gamma(\beta) t^{\alpha-1}}{\Delta}, \quad \forall(t, s) \in J \times J .
$$

Furthermore,

$$
0 \leq \frac{K_{1}(t, s)}{1+t^{\alpha+\beta-1}} \leq \frac{\Gamma(\beta)}{\Delta}, \quad \forall(t, s) \in J \times J .
$$

By a similar calculation, we can prove other inequality results about $K_{2}(t, s), K_{3}(t, s)$, and $K_{4}(t, s)$. So the proof is completed.

Remark 2.6 From (2.5), (2.8), and (2.10), by a direct calculation, we have

$$
\left\{\begin{array}{l}
D^{\alpha-1} u(t)=\int_{0}^{+\infty} H_{1}(t, s) x(s) \mathrm{d} s+\int_{0}^{+\infty} H_{3}(t, s) y(s) \mathrm{d} s \\
D^{\beta-1} v(t)=\int_{0}^{+\infty} H_{2}(t, s) y(s) \mathrm{d} s+\int_{0}^{+\infty} H_{4}(t, s) x(s) \mathrm{d} s
\end{array}\right.
$$

where

$$
\begin{aligned}
& H_{1}(t, s)=H_{11}(t, s)+H_{12}(t, s), \quad H_{2}(t, s)=H_{11}(t, s)+H_{22}(t, s), \\
& H_{3}(t, s)=\frac{\Gamma(\alpha) \Gamma(\beta)}{\Delta} \int_{0}^{+\infty} h(t) K_{21}(t, s) \mathrm{d} t, \\
& H_{4}(t, s)=\frac{\Gamma(\alpha) \Gamma(\beta)}{\Delta} \int_{0}^{+\infty} g(t) K_{11}(t, s) \mathrm{d} t
\end{aligned}
$$

with

$$
\begin{aligned}
& H_{11}(t, s)=\left\{\begin{array}{ll}
0, & 0 \leq s \leq t \leq+\infty, \\
1, & 0 \leq t \leq s \leq+\infty,
\end{array} \quad H_{12}(t, s)=\frac{\Upsilon_{1} \Gamma(\alpha)}{\Delta} \int_{0}^{+\infty} g(t) K_{11}(t, s) \mathrm{d} t .\right. \\
& H_{22}(t, s)=\frac{\Upsilon_{2} \Gamma(\beta)}{\Delta} \int_{0}^{+\infty} h(t) K_{21}(t, s) \mathrm{d} t .
\end{aligned}
$$

Remark 2.7 From Lemma 2.5, by a direct calculation, we can easily obtain that

$$
0 \leq H_{1}(t, s)=H_{11}(t, s)+H_{12}(t, s) \leq 1+\frac{\Upsilon_{1} \Upsilon_{2}}{\Delta}=\frac{\Gamma(\alpha) \Gamma(\beta)}{\Delta}, \quad \forall(t, s) \in J \times J,
$$




$$
\begin{aligned}
& 0 \leq H_{2}(t, s)=H_{11}(t, s)+H_{22}(t, s) \leq 1+\frac{\Upsilon_{1} \Upsilon_{2}}{\Delta}=\frac{\Gamma(\alpha) \Gamma(\beta)}{\Delta}, \quad \forall(t, s) \in J \times J, \\
& 0 \leq H_{3}(t, s) \leq \frac{\Gamma(\alpha) \Upsilon_{1}}{\Delta}, \quad \forall(t, s) \in J \times J, \\
& 0 \leq H_{4}(t, s) \leq \frac{\Gamma(\beta) \Upsilon_{2}}{\Delta}, \quad \forall(t, s) \in J \times J .
\end{aligned}
$$

Define two spaces

$$
\begin{aligned}
& X=\left\{u \in C(J), D^{\alpha-1} u \in C(J)\left|\sup _{t \in J} \frac{|u(t)|}{1+t^{\alpha+\beta-1}}<+\infty, \sup _{t \in J}\right| D^{\alpha-1} u(t) \mid<+\infty\right\}, \\
& Y=\left\{v \in C(J), D^{\beta-1} v \in C(J)\left|\sup _{t \in J} \frac{|v(t)|}{1+t^{\alpha+\beta-1}}<+\infty, \sup _{t \in J}\right| D^{\beta-1} v(t) \mid<+\infty\right\},
\end{aligned}
$$

equipped with the norms

$$
\begin{aligned}
& \|u\|_{X}=\max \left\{\sup _{t \in J} \frac{|u(t)|}{1+t^{\alpha+\beta-1}}, \sup _{t \in J}\left|D^{\alpha-1} u(t)\right|\right\}, \\
& \|v\|_{Y}=\max \left\{\sup _{t \in J} \frac{|v(t)|}{1+t^{\alpha+\beta-1}}, \sup _{t \in J}\left|D^{\beta-1} v(t)\right|\right\},
\end{aligned}
$$

where $2<\alpha, \beta \leq 3$. $C(J)$ denotes the space of all continuous functions defined on $[0,+\infty)$.

Lemma $2.8\left(X,\|\cdot\|_{X}\right)$ and $\left(Y,\|\cdot\|_{Y}\right)$ are two Banach spaces.

Proof The proof is similar to that of Lemma 2.4 in [29], so we omit it.

Lemma 2.9 (see [44]) Let $U \subset X$ be a bounded set. Then $U$ is relatively compact in $X$ if the following conditions hold:

(i) For any $u \in U, \frac{u(t)}{1+t^{\alpha-1}}$ and $D^{\alpha-1} u(t)$ are equicontinuous on any compact interval of $)$;

(ii) For any $\varepsilon>0$, there is a constant $C=C(\varepsilon)>0$ such that $\left|\frac{u\left(t_{1}\right)}{1+t_{1}^{\alpha-1}}-\frac{u\left(t_{2}\right)}{1+t_{2}^{\alpha-1}}\right|<\varepsilon$ and $\left|D^{\alpha-1} u\left(t_{1}\right)-D^{\alpha-1} u\left(t_{2}\right)\right|<\varepsilon$ for any $t_{1}, t_{2} \geq C$ and $u \in U$.

Remark 2.10 Let $U \subset X$ be a bounded set. According to Lemmas 2.8 and 2.9, it is clear that $U$ is relatively compact in $\mathrm{X}$ if the following conditions hold:

(i) For any $u \in U, \frac{u(t)}{1+t^{\alpha+\beta-1}}$ and $D^{\alpha-1} u(t)$ are equicontinuous on any compact interval of $J$;

(ii) For any $\varepsilon>0$, there is a constant $C=C(\varepsilon)>0$ such that $\left|\frac{u\left(t_{1}\right)}{1+t_{1}^{\alpha+\beta-1}}-\frac{u\left(t_{2}\right)}{1+t_{2}^{\alpha+\beta-1}}\right|<\varepsilon$ and $\left|D^{\alpha-1} u\left(t_{1}\right)-D^{\alpha-1} u\left(t_{2}\right)\right|<\varepsilon$ for any $t_{1}, t_{2} \geq C$ and $u \in U$.

\section{Main results}

We define the cone $P \subset X \times Y$ as $P=\left\{(u, v) \in X \times Y \mid u(t) \geq 0, v(t) \geq 0, D^{\alpha-1} u(t) \geq\right.$ $\left.0, D^{\beta-1} v(t) \geq 0, t \in J\right\}$. From Lemma 2.4 it is easy to know that the fractional differential system (1.6) is equivalent to the following system of Hammerstein-type integral equations:

$$
\begin{aligned}
\left(\begin{array}{l}
u(t) \\
v(t)
\end{array}\right) & =\left(\begin{array}{l}
\int_{0}^{+\infty} K_{1}(t, s) \varphi_{(u, v)}(s) \mathrm{d} s+\int_{0}^{+\infty} K_{3}(t, s) \psi_{(u, v)}(s) \mathrm{d} s \\
\int_{0}^{+\infty} K_{2}(t, s) \psi_{(u, v)}(s) \mathrm{d} s+\int_{0}^{+\infty} K_{4}(t, s) \varphi_{(u, v)}(s) \mathrm{d} s
\end{array}\right) \\
& :=\left(\begin{array}{l}
\digamma_{1}(u, v)(t) \\
\digamma_{2}(u, v)(t)
\end{array}\right) \quad \text { for } u, v \in P, t \in J,
\end{aligned}
$$


and for convenience, we set

$$
\left\{\begin{array}{l}
\varphi_{(u, v)}(s)=\varphi\left(s, u(s), v(s), D^{\beta-1} v(s)\right) \\
\psi_{(u, v)}(s)=\psi\left(s, u(s), v(s), D^{\alpha-1} u(s)\right) .
\end{array}\right.
$$

Then we can define an operator $\digamma: P \times P \rightarrow P \times P$ as follows:

$$
\digamma(u, v)(t)=\left(\digamma_{1}, \digamma_{2}\right)(u, v)(t) \quad \text { for } u, v \in P, t \in J .
$$

Therefore, if $(u, v) \in(P \times P) \backslash\{\mathbf{0}\}$ is a fixed point of $\digamma$, then $(u, v)$ is a positive solution for the fractional differential system (1.6). Next, we will directly study the existence of fixed points of the operator $\digamma$.

By Remark 2.6 and (3.1), we have

$$
\begin{aligned}
& \left(\begin{array}{l}
D^{\alpha-1} \digamma_{1}(u, v)(t) \\
D^{\beta-1} \digamma_{2}(u, v)(t)
\end{array}\right) \\
& \quad=\left(\begin{array}{l}
\int_{0}^{+\infty} H_{1}(t, s) \varphi_{(u, v)}(s) \mathrm{d} s+\int_{0}^{+\infty} H_{3}(t, s) \psi_{(u, v)}(s) \mathrm{d} s \\
\int_{0}^{+\infty} H_{2}(t, s) \psi_{(u, v)}(s) \mathrm{d} s+\int_{0}^{+\infty} H_{4}(t, s) \varphi_{(u, v)}(s) \mathrm{d} s
\end{array}\right) \quad \text { for } u, v \in P, t \in J .
\end{aligned}
$$

Lemma 3.1 If assumptions (C0) and (C2) are satisfied, then

$$
\int_{0}^{+\infty}\left|\varphi_{(u, v)}(s)\right| \mathrm{d} s \leq a_{0}^{*}+\sum_{k=1}^{3} a_{k}^{*}\|(u, v)\|_{X \times Y}^{\lambda_{k}}, \quad \forall(u, v) \in X \times Y,
$$

and

$$
\int_{0}^{+\infty}\left|\psi_{(u, v)}(s)\right| \mathrm{d} s \leq b_{0}^{*}+\sum_{k=1}^{3} b_{k}^{*}\|(u, v)\|_{X \times Y}^{\tau_{k}}, \quad \forall(u, v) \in X \times Y .
$$

Proof For $\forall(u, v) \in X \times Y$, by assumptions (C0) and (C2), we have

$$
\begin{aligned}
\int_{0}^{+\infty} & \left|\varphi_{(u, v)}(s)\right| \mathrm{d} s \\
\leq & \int_{0}^{+\infty}\left(a_{0}(s)+a_{1}(s)|u(s)|^{\lambda_{1}}+a_{2}(s)|v(s)|^{\lambda_{2}}+a_{3}(s)\left|D^{\beta-1} v(s)\right|^{\lambda_{3}}\right) \mathrm{d} s \\
\leq & \left.a_{0}^{*}+\int_{0}^{+\infty} a_{1}(s)\right)\left(1+s^{\alpha+\beta-1}\right)^{\lambda_{1}} \frac{|u(s)|^{\lambda_{1}}}{\left(1+s^{\alpha+\beta-1}\right)^{\lambda_{1}}} \mathrm{~d} s \\
& \left.+\int_{0}^{+\infty} a_{2}(s)\right)\left(1+s^{\alpha+\beta-1}\right)^{\lambda_{2}} \frac{|v(s)|^{\lambda_{2}}}{\left(1+s^{\alpha+\beta-1}\right)^{\lambda_{2}}} d s+\int_{0}^{+\infty} a_{3}(s)\left|D^{\beta-1} v(s)\right|^{\lambda_{3}} \mathrm{~d} s \\
\leq & a_{0}^{*}+a_{1}^{*}\|u\|_{X}^{\lambda_{1}}+a_{2}^{*}\|v\|_{Y}^{\lambda_{2}}+a_{3}^{*}\|v\|_{Y}^{\lambda_{3}} \\
\leq & a_{0}^{*}+\sum_{k=1}^{3} a_{k}^{*}\|(u, v)\|_{X \times Y}^{\lambda_{k}}
\end{aligned}
$$


and

$$
\begin{aligned}
\int_{0}^{+\infty} & \left|\psi_{(u, v)}(s)\right| \mathrm{d} s \\
\leq & \int_{0}^{+\infty}\left(b_{0}(s)+b_{1}(s)|u(s)|^{\tau_{1}}+b_{2}(s)|v(s)|^{\tau_{2}}+b_{3}(s)\left|D^{\alpha-1} u(s)\right|^{\tau_{3}}\right) \mathrm{d} s \\
\leq & \left.b_{0}^{*}+\int_{0}^{+\infty} b_{1}(s)\right)\left(1+s^{\alpha+\beta-1}\right)^{\tau_{1}} \frac{|u(s)|^{\tau_{1}}}{\left(1+s^{\alpha+\beta-1}\right)^{\tau_{1}}} \mathrm{~d} s \\
& \left.+\int_{0}^{+\infty} b_{2}(s)\right)\left(1+s^{\alpha+\beta-1}\right)^{\tau_{2}} \frac{|v(s)|^{\tau_{2}}}{\left(1+s^{\alpha+\beta-1}\right)^{\tau_{2}}} d s+\int_{0}^{+\infty} b_{3}(s)\left|D^{\alpha-1} u(s)\right|^{\tau_{3}} \mathrm{~d} s \\
\leq & b_{0}^{*}+b_{1}^{*}\|u\|_{X}^{\tau_{1}}+b_{2}^{*}\|v\|_{Y}^{\tau_{2}}+b_{3}^{*}\|u\|_{X}^{\tau_{3}} \\
\leq & b_{0}^{*}+\sum_{k=1}^{3} b_{k}^{*}\|(u, v)\|_{X \times Y}^{\tau_{k}} .
\end{aligned}
$$

Lemma 3.2 If assumptions (C0), (C1), and (C2) are satisfied, then the operator $\digamma: P \rightarrow P$ is completely continuous.

Proof Since $K_{i}(t, s) \geq 0, \forall(t, s) \in J \times J, i=1,2,3,4$, and $\varphi \geq 0, \psi \geq 0, \forall(u, v) \in P \times P$, we have $\digamma_{1}(u, v)(t) \geq 0, \digamma_{2}(u, v)(t) \geq 0, \forall(u, v) \in P, t \in J$. So it is obvious that $\digamma: P \rightarrow P$.

Next we show that the operator $\digamma: P \rightarrow P$ is relatively compact. First let $U=\{(u, v) \mid$ $\left.(u, v) \in P,\|(u, v)\|_{X \times Y} \leq M\right\}$. For $\forall(u, v) \in U$, by Lemma 2.4, Lemma 2.5, and Lemma 3.1, we have

$$
\begin{aligned}
\sup _{t \in J} & \frac{\left|\digamma_{1}(u, v)(t)\right|}{1+t^{\alpha+\beta-1}} \\
& \leq \sup _{t \in J}\left|\int_{0}^{+\infty} \frac{K_{1}(t, s)}{1+t^{\alpha+\beta-1}} \varphi_{(u, v)}(s) \mathrm{d} s\right|+\sup _{t \in J}\left|\int_{0}^{+\infty} \frac{K_{3}(t, s)}{1+t^{\alpha+\beta-1}} \psi_{(u, v)}(s) \mathrm{d} s\right| \\
& \leq \frac{\Gamma(\beta)}{\Delta} \int_{0}^{+\infty}\left|\varphi_{(u, v)}(s)\right| \mathrm{d} s+\frac{\Upsilon_{1}}{\Delta} \int_{0}^{+\infty}\left|\psi_{(u, v)}(s)\right| \mathrm{d} s \\
& \leq \frac{\Gamma(\beta)+\Upsilon_{1}}{\Delta}\left[a_{0}^{*}+\sum_{k=1}^{3} a_{k}^{*}\|(u, v)\|_{X \times Y}^{\lambda_{k}}+b_{0}^{*}+\sum_{k=1}^{3} b_{k}^{*}\|(u, v)\|_{X \times Y}^{\tau_{k}}\right] \\
& \leq \frac{\Gamma(\beta)+\Upsilon_{1}}{\Delta}\left[a_{0}^{*}+b_{0}^{*}+\sum_{k=1}^{3}\left(a_{k}^{*} M^{\lambda_{k}}+b_{k}^{*} M^{\tau_{k}}\right)\right]
\end{aligned}
$$

By Remark 2.6, Remark 2.7, and Lemma 3.1, we have

$$
\begin{aligned}
& \sup _{t \in J}\left|D^{\alpha-1} \digamma_{1}(u, v)(t)\right| \\
& \leq \sup _{t \in J}\left|\int_{0}^{\infty} H_{1}(t, s) \varphi_{(u, v)}(s) \mathrm{d} s\right|+\sup _{t \in J}\left|\int_{0}^{\infty} H_{3}(t, s) \psi_{(u, v)}(s) \mathrm{d} s\right| \\
& \leq \frac{\Gamma(\alpha) \Gamma(\beta)}{\Delta} \int_{0}^{+\infty}\left|\varphi_{(u, v)}(s)\right| \mathrm{d} s+\frac{\Gamma(\alpha) \Upsilon_{1}}{\Delta} \int_{0}^{+\infty}\left|\psi_{(u, v)}(s)\right| \mathrm{d} s \\
& \leq \frac{\Gamma(\alpha)\left(\Gamma(\beta)+\Upsilon_{1}\right)}{\Delta}\left[a_{0}^{*}+\sum_{k=1}^{3} a_{k}^{*}\|(u, v)\|_{X \times Y}^{\lambda_{k}}+b_{0}^{*}+\sum_{k=1}^{3} b_{k}^{*}\|(u, v)\|_{X \times Y}^{\tau_{k}}\right]
\end{aligned}
$$




$$
\leq \frac{\Gamma(\alpha)\left(\Gamma(\beta)+\Upsilon_{1}\right)}{\Delta}\left[a_{0}^{*}+b_{0}^{*}+\sum_{k=1}^{3}\left(a_{k}^{*} M^{\lambda_{k}}+b_{k}^{*} M^{\tau_{k}}\right)\right]
$$

Thus

$$
\begin{aligned}
& \left\|\digamma_{1}(u, v)\right\|_{X} \\
& \quad=\max \left\{\sup _{t \in J} \frac{\left|\digamma_{1}(u, v)(t)\right|}{1+t^{\alpha+\beta-1}}, \sup _{t \in J}\left|D^{\alpha-1} \digamma_{1}(u, v)(t)\right|\right\} \\
& \leq \frac{\max \left\{\Gamma(\beta)+\Upsilon_{1}, \Gamma(\alpha)\left(\Gamma(\beta)+\Upsilon_{1}\right)\right\}}{\Delta}\left[a_{0}^{*}+b_{0}^{*}+\sum_{k=1}^{3}\left(a_{k}^{*} M^{\lambda_{k}}+b_{k}^{*} M^{\tau_{k}}\right)\right] .
\end{aligned}
$$

Similarly

$$
\left\|\digamma_{2}(u, v)\right\|_{Y} \leq \frac{\max \left\{\Gamma(\alpha)+\Upsilon_{2}, \Gamma(\beta)\left(\Gamma(\alpha)+\Upsilon_{2}\right)\right\}}{\Delta}\left[a_{0}^{*}+b_{0}^{*}+\sum_{k=1}^{3}\left(a_{k}^{*} M^{\lambda_{k}}+b_{k}^{*} M^{\tau_{k}}\right)\right] .
$$

Therefore

$$
\|\digamma(u, v)\|_{X \times Y}=\max \left\{\left\|\digamma_{1}(u, v)\right\|_{X},\left\|\digamma_{2}(u, v)\right\|_{Y}\right\} \leq \frac{\Theta}{\Delta}\left[a_{0}^{*}+b_{0}^{*}+\sum_{k=1}^{3}\left(a_{k}^{*} M^{\lambda_{k}}+b_{k}^{*} M^{\tau_{k}}\right)\right],
$$

which implies that $T U$ is uniformly bounded.

Second, let $I \subset J$ be any compact interval. Then, for all $t_{1}, t_{2} \in I, t_{2}>t_{1}$ and $(u, v) \in U$, we have

$$
\begin{aligned}
& \left|\frac{\digamma_{1}(u, v)\left(t_{2}\right)}{1+t_{2}^{\alpha+\beta-1}}-\frac{\digamma_{1}(u, v)\left(t_{1}\right)}{1+t_{1}^{\alpha+\beta-1}}\right| \\
& \quad \leq \int_{0}^{+\infty}\left|\frac{K_{1}\left(t_{2}, s\right)}{1+t_{2}^{\alpha+\beta-1}}-\frac{K_{1}\left(t_{1}, s\right)}{1+t_{1}^{\alpha+\beta-1}}\right|\left|\varphi_{(u, v)}(s)\right| \mathrm{d} s \\
& \quad+\int_{0}^{+\infty}\left|\frac{K_{3}\left(t_{2}, s\right)}{1+t_{2}^{\alpha+\beta-1}}-\frac{K_{3}\left(t_{1}, s\right)}{1+t_{1}^{\alpha+\beta-1}}\right|\left|\psi_{(u, v)}(s)\right| \mathrm{d} s .
\end{aligned}
$$

Notice that $K_{1}(t, s) /\left(1+t^{\alpha+\beta-1}\right), K_{3}(t, s) /\left(1+t^{\alpha+\beta-1}\right)$ are uniformly continuous for any $(t, s) \in$ $I \times I$. Furthermore, $K_{1}(t, s) /\left(1+t^{\alpha+\beta-1}\right), K_{3}(t, s) /\left(1+t^{\alpha+\beta-1}\right)$ only depend on $t$ for $s \geq t$, which implies that $K_{1}(t, s) /\left(1+t^{\alpha+\beta-1}\right), K_{3}(t, s) /\left(1+t^{\alpha+\beta-1}\right)$ are uniformly continuous on $I \times(J \backslash I)$. Thus, for all $s \in J$ and $t_{1}, t_{2} \in I$, we have

$$
\begin{aligned}
& \forall \epsilon>0, \exists \delta(\epsilon) \text { such that if }\left|t_{1}-t_{2}\right|<\delta \text {, then } \\
& \left|\frac{K_{1}\left(t_{2}, s\right)}{1+t_{2}^{\alpha+\beta-1}}-\frac{K_{1}\left(t_{1}, s\right)}{1+t_{1}^{\alpha+\beta-1}}\right|<\epsilon, \quad\left|\frac{K_{3}\left(t_{2}, s\right)}{1+t_{2}^{\alpha+\beta-1}}-\frac{K_{3}\left(t_{1}, s\right)}{1+t_{1}^{\alpha+\beta-1}}\right|<\epsilon .
\end{aligned}
$$

Combining (3.6) and (3.7) with Lemma 3.1, for all $s \in J,(u, v) \in U$, and $t_{1}, t_{2} \in I$, we have

$$
\left|\frac{\digamma_{1}(u, v)\left(t_{2}\right)}{1+t_{2}^{\alpha+\beta-1}}-\frac{\digamma_{1}(u, v)\left(t_{1}\right)}{1+t_{1}^{\alpha+\beta-1}}\right| \leq\left[a_{0}^{*}+\sum_{k=1}^{3} a_{k}^{*} M^{\lambda_{k}}+b_{0}^{*}+\sum_{k=1}^{3} b_{k}^{*} M^{\tau_{k}}\right] \epsilon,
$$

which implies that $\digamma_{1}(u, v)(t) /\left(1+t^{\alpha+\beta-1}\right)$ is equicontinuous on $I$. 
Note that

$$
D^{\alpha-1} \digamma_{1}(u, v)(t)=\int_{0}^{+\infty} H_{1}(t, s) \varphi_{(u, v)}(s) \mathrm{d} s+\int_{0}^{+\infty} H_{3}(t, s) \psi_{(u, v)}(s) \mathrm{d} s
$$

and $H_{1}(t, s), H_{3}(t, s) \in C(J \times J)$ do not depend on $t$, which infers that $D^{\alpha-1} \digamma_{1}(u, v)(t)$ is equicontinuous on $I$. In the same way, we can show that $\digamma_{2}(u, v)(t) /\left(1+t^{\alpha+\beta-1}\right)$ and $D^{\beta-1} \digamma_{2}(u, v)(t)$ are equicontinuous. Thus condition (i) of Remark 2.10 is satisfied.

Then we show that operators $\digamma_{1}, \digamma_{2}$ are equiconvergent at $+\infty$. Since

$$
\lim _{t \rightarrow+\infty} \frac{K_{1}(t, s)}{1+t^{\alpha+\beta-1}}=0, \quad \lim _{t \rightarrow+\infty} \frac{K_{3}(t, s)}{1+t^{\alpha+\beta-1}}=0,
$$

we can infer that, for any $\epsilon>0$, there exists a sufficiently large constant $C=C(\epsilon)>0$, for any $t_{1}, t_{2} \geq C$ and $s \in J$, such that

$$
\left|\frac{K_{1}\left(t_{2}, s\right)}{1+t_{2}^{\alpha+\beta-1}}-\frac{K_{1}\left(t_{1}, s\right)}{1+t_{1}^{\alpha+\beta-1}}\right|<\epsilon, \quad\left|\frac{K_{3}\left(t_{2}, s\right)}{1+t_{2}^{\alpha+\beta-1}}-\frac{K_{3}\left(t_{1}, s\right)}{1+t_{1}^{\alpha+\beta-1}}\right|<\epsilon
$$

Therefore, by Lemma 3.1 and (3.6), we conclude that $\digamma_{1}(u, v)(t) / 1+t^{\alpha+\beta-1}$ is equiconvergent at $+\infty$. On the other hand, the functions $H_{1}(t, s), H_{3}(t, s)$ do not depend on $t$, it is obvious that $D^{\alpha-1} \digamma_{1}(u, v)(t)$ is equiconvergent at $+\infty$. Similarly, $\digamma_{2}(u, v)(t) / 1+t^{\alpha+\beta-1}$ and $D^{\alpha-1} \digamma_{2}(u, v)(t)$ are equiconvergent at $+\infty$. Thus condition (ii) of Remark 2.10 is satisfied.

As can be seen from the above discussion, all the conditions of Remark 2.10 are satisfied. Thus the operator $\digamma: P \rightarrow P$ is relatively compact.

Finally, we prove that the operator $\digamma: P \rightarrow P$ is continuous. Let $\left(u_{n}, v_{n}\right),(u, v) \in P$ such that $\left(u_{n}, v_{n}\right) \rightarrow(u, v)(n \rightarrow \infty)$. Then $\left\|\left(u_{n}, v_{n}\right)\right\|_{X \times Y}<+\infty,\|(u, v)\|_{X \times Y}<+\infty$. Similar to (3.3) and (3.4), we can obtain

$$
\begin{aligned}
\sup _{t \in J} & \frac{\left|\digamma_{1}\left(u_{n}, v_{n}\right)(t)\right|}{1+t^{\alpha+\beta-1}} \\
\leq & \frac{\Gamma(\beta)+\Upsilon_{1}}{\Delta}\left[a_{0}^{*}+b_{0}^{*}+\sum_{k=1}^{3}\left(a_{k}^{*}\left\|\left(u_{n}, v_{n}\right)\right\|_{X \times Y}^{\lambda_{k}}+b_{k}^{*}\left\|\left(u_{n}, v_{n}\right)\right\|_{X \times Y}^{\tau_{k}}\right)\right]<+\infty
\end{aligned}
$$

and

$$
\begin{aligned}
& \sup _{t \in J}\left|D^{\alpha-1} \digamma_{1}\left(u_{n}, v_{n}\right)(t)\right| \\
& \leq \frac{\Gamma(\alpha)\left(\Gamma(\beta)+\Upsilon_{1}\right)}{\Delta}\left[a_{0}^{*}+b_{0}^{*}+\sum_{k=1}^{3}\left(a_{k}^{*}\left\|\left(u_{n}, v_{n}\right)\right\|_{X \times Y}^{\lambda_{k}}+b_{k}^{*}\left\|\left(u_{n}, v_{n}\right)\right\|_{X \times Y}^{\tau_{k}}\right)\right]<+\infty .
\end{aligned}
$$

By the continuity of function $\varphi, \psi$ and the Lebesgue dominated convergence theorem, we have

$$
\begin{aligned}
& \lim _{n \rightarrow \infty} \frac{\digamma_{1}\left(u_{n}, v_{n}\right)(t)}{1+t^{\alpha+\beta-1}} \\
& \quad=\lim _{n \rightarrow \infty}\left[\int_{0}^{+\infty} \frac{K_{1}(t, s)}{1+t^{\alpha+\beta-1}} \varphi_{\left(u_{n}, v_{n}\right)}(s) \mathrm{d} s+\int_{0}^{+\infty} \frac{K_{3}(t, s)}{1+t^{\alpha+\beta-1}} \psi_{\left(u_{n}, v_{n}\right)}(s) \mathrm{d} s\right] \\
& \quad=\int_{0}^{+\infty} \frac{K_{1}(t, s)}{1+t^{\alpha+\beta-1}} \varphi_{(u, v)}(s) \mathrm{d} s+\int_{0}^{+\infty} \frac{K_{3}(t, s)}{1+t^{\alpha+\beta-1}} \psi_{(u, v)}(s) \mathrm{d} s=\frac{\digamma_{1}(u, v)(t)}{1+t^{\alpha+\beta-1}}
\end{aligned}
$$


and

$$
\begin{aligned}
& \lim _{n \rightarrow \infty} D^{\alpha-1} \digamma_{1}\left(u_{n}, v_{n}\right)(t) \\
& \quad=\lim _{n \rightarrow \infty}\left[\int_{0}^{+\infty} H_{1}(t, s) \varphi_{\left(u_{n}, v_{n}\right)}(s) \mathrm{d} s+\int_{0}^{+\infty} H_{3}(t, s) \psi_{\left(u_{n}, v_{n}\right)}(s) \mathrm{d} s\right] \\
& \quad=\int_{0}^{\infty} H_{1}(t, s) \varphi_{(u, v)}(s) \mathrm{d} s+\int_{0}^{+\infty} H_{3}(t, s) \psi_{(u, v)}(s) \mathrm{d} s=D^{\alpha-1} \digamma_{1}(u, v)(t) .
\end{aligned}
$$

Then, as $n \rightarrow \infty$,

$$
\begin{aligned}
\sup _{t \in J} & \frac{\left|\digamma_{1}\left(u_{n}, v_{n}\right)(t)-\digamma_{1}(u, v)(t)\right|}{1+t^{\alpha+\beta-1}} \\
\leq & \sup _{t \in J} \int_{0}^{+\infty} \frac{K_{1}(t, s)}{1+t^{\alpha+\beta-1}}\left|\varphi_{\left(u_{n}, v_{n}\right)}(s)-\varphi_{(u, v)}(s)\right| \mathrm{d} s \\
& \quad+\sup _{t \in J} \int_{0}^{+\infty} \frac{K_{3}(t, s)}{1+t^{\alpha+\beta-1}}\left|\psi_{\left(u_{n}, v_{n}\right)}(s)-\psi_{(u, v)}(s)\right| \mathrm{d} s \\
\leq & \frac{\Gamma(\beta)+\Upsilon_{1}}{\Delta}\left[\int_{0}^{+\infty}\left|\varphi_{\left(u_{n}, v_{n}\right)}(s)-\varphi_{(u, v)}(s)\right| \mathrm{d} s+\int_{0}^{+\infty}\left|\psi_{\left(u_{n}, v_{n}\right)}(s)-\psi_{(u, v)}(s)\right| \mathrm{d} s\right] \\
& \rightarrow 0
\end{aligned}
$$

and as $n \rightarrow \infty$,

$$
\begin{aligned}
& \sup _{t \in J}\left|D^{\alpha-1} \digamma_{1}\left(u_{n}, v_{n}\right)(t)-D^{\alpha-1} \digamma_{1}(u, v)(t)\right| \\
& \leq \sup _{t \in J} \int_{0}^{+\infty} H_{1}(t, s)\left|\varphi_{\left(u_{n}, v_{n}\right)}(s)-\varphi_{(u, v)}(s)\right| \mathrm{d} s \\
& \quad+\sup _{t \in J} \int_{0}^{+\infty} H_{3}(t, s)\left|\psi_{\left(u_{n}, v_{n}\right)}(s)-\varphi_{(u, v)}(s)\right| \mathrm{d} s \\
& \leq \frac{\Gamma(\alpha)\left(\Gamma(\beta)+\Upsilon_{1}\right)}{\Delta}\left[\int_{0}^{+\infty}\left|\varphi_{\left(u_{n}, v_{n}\right)}(s)-\varphi_{(u, v)}(s)\right| \mathrm{d} s+\int_{0}^{+\infty}\left|\psi_{\left(u_{n}, v_{n}\right)}(s)-\varphi_{(u, v)}(s)\right| \mathrm{d} s\right] \\
& \rightarrow 0 .
\end{aligned}
$$

So, as $n \rightarrow \infty$,

$$
\begin{aligned}
& \left\|\digamma_{1}\left(u_{n}, v_{n}\right)-\digamma_{1}(u, v)\right\|_{X} \\
& \quad=\max \left\{\sup _{t \in J} \frac{\left|\digamma_{1}\left(u_{n}, v_{n}\right)(t)-\digamma_{1}(u, v)(t)\right|}{1+t^{\alpha+\beta-1}}, \sup _{t \in J}\left|D^{\alpha-1} \digamma_{1}\left(u_{n}, v_{n}\right)(t)-D^{\alpha-1} \digamma_{1}(u, v)(t)\right|\right\} \\
& \quad \rightarrow 0
\end{aligned}
$$

which implies that the operator $\digamma_{1}$ is continuous. By the same way, we can obtain that the operator $\digamma_{2}$ is continuous. That is, the operator $\digamma$ is continuous.

In view of all above arguments, the operator $\digamma: P \rightarrow P$ is completely continuous. So the proof is completed. 
Define a partial order over the product space:

$$
\left(\begin{array}{l}
u_{1} \\
v_{1}
\end{array}\right) \geq\left(\begin{array}{l}
u_{2} \\
v_{2}
\end{array}\right)
$$

if $u_{1}(t) \geq u_{2}(t), v_{1}(t) \geq v_{2}(t), D^{\alpha-1} u_{1}(t) \geq D^{\alpha-1} u_{2}(t), D^{\beta-1} v_{1}(t) \geq D^{\beta-1} v_{2}(t), t \in J$.

Theorem 3.3 Suppose that (C0), (C1), (C2), and (C3) are satisfied. Then there exists a positive constant $R$ such that system (1.6) has two positive solutions $\left(u^{*}, v^{*}\right)$ and $\left(w^{*}, z^{*}\right)$ satisfying $0 \leq\left\|\left(u^{*}, v^{*}\right)\right\|_{X \times Y} \leq R$ and $0 \leq\left\|\left(w^{*}, z^{*}\right)\right\|_{X \times Y} \leq R$ with $\lim _{n \rightarrow \infty}\left(u_{n}, v_{n}\right)=\left(u^{*}, v^{*}\right)$ and $\lim _{n \rightarrow \infty}\left(w_{n}, z_{n}\right)=\left(w^{*}, z^{*}\right)$, where $\left(u_{n}, v_{n}\right)$ and $\left(w_{n}, z_{n}\right)$ can be given by the following monotone iterative sequences:

$$
\left(\begin{array}{l}
u_{n}(t) \\
v_{n}(t)
\end{array}\right)=\left(\begin{array}{l}
\digamma_{1}\left(u_{n-1}, v_{n-1}\right)(t) \\
\digamma_{2}\left(u_{n-1}, v_{n-1}\right)(t)
\end{array}\right), \quad n=1,2, \ldots, \text { with }\left(\begin{array}{l}
u_{0}(t) \\
v_{0}(t)
\end{array}\right)=\left(\begin{array}{l}
R t^{\alpha} \\
R t^{\beta}
\end{array}\right)
$$

and

$$
\left(\begin{array}{l}
w_{n}(t) \\
z_{n}(t)
\end{array}\right)=\left(\begin{array}{l}
\digamma_{1}\left(w_{n-1}, z_{n-1}\right)(t) \\
\digamma_{2}\left(w_{n-1}, z_{n-1}\right)(t)
\end{array}\right), \quad n=1,2, \ldots, \text { with }\left(\begin{array}{l}
w_{0}(t) \\
z_{0}(t)
\end{array}\right)=\left(\begin{array}{l}
0 \\
0
\end{array}\right) .
$$

In addition,

$$
\begin{aligned}
\left(\begin{array}{c}
w_{0}(t) \\
z_{0}(t)
\end{array}\right) & \leq\left(\begin{array}{c}
w_{1}(t) \\
z_{1}(t)
\end{array}\right) \leq \cdots \leq\left(\begin{array}{c}
w_{n}(t) \\
z_{n}(t)
\end{array}\right) \leq \cdots \leq\left(\begin{array}{c}
w^{*}(t) \\
z^{*}(t)
\end{array}\right) \leq \cdots \leq\left(\begin{array}{c}
u^{*}(t) \\
v^{*}(t)
\end{array}\right) \\
& \leq \cdots \leq\left(\begin{array}{l}
u_{n}(t) \\
v_{n}(t)
\end{array}\right) \leq \cdots \leq\left(\begin{array}{c}
u_{2}(t) \\
v_{2}(t)
\end{array}\right) \leq\left(\begin{array}{c}
u_{1}(t) \\
v_{1}(t)
\end{array}\right) \leq\left(\begin{array}{c}
u_{0}(t) \\
v_{0}(t)
\end{array}\right)
\end{aligned}
$$

and

$$
\begin{aligned}
\left(\begin{array}{c}
D^{\alpha-1} w_{0}(t) \\
D^{\beta-1} z_{0}(t)
\end{array}\right) & \leq\left(\begin{array}{c}
D^{\alpha-1} w_{1}(t) \\
D^{\beta-1} z_{1}(t)
\end{array}\right) \leq \cdots \leq\left(\begin{array}{c}
D^{\alpha-1} w_{n}(t) \\
D^{\beta-1} z_{n}(t)
\end{array}\right) \leq \cdots \leq\left(\begin{array}{c}
D^{\alpha-1} w^{*}(t) \\
D^{\beta-1} z^{*}(t)
\end{array}\right) \\
& \leq \cdots \leq\left(\begin{array}{c}
D^{\alpha_{1}-1} u^{*}(t) \\
D^{\alpha 2} v^{*}(t)
\end{array}\right) \leq \cdots \leq\left(\begin{array}{c}
D^{\alpha-1} u_{n}(t) \\
D^{\beta-1} v_{n}(t)
\end{array}\right) \leq \cdots \leq\left(\begin{array}{c}
D^{\alpha-1} u_{2}(t) \\
D^{\beta-1} v_{2}(t)
\end{array}\right) \\
& \leq\left(\begin{array}{c}
D^{\alpha-1} u_{1}(t) \\
D^{\beta-1} v_{1}(t)
\end{array}\right) \leq\left(\begin{array}{c}
D^{\alpha-1} u_{0}(t) \\
D^{\beta-1} v_{0}(t)
\end{array}\right) .
\end{aligned}
$$

Proof First, Lemma 3.2 brings about the fact that $\digamma(P) \subset P$ for any $(u, v) \in P, t \in J$.

Next, let

$$
R \geq \max \left\{8 \Lambda a_{0}^{*}, 8 \Lambda b_{0}^{*},\left(8 \Lambda a_{k}^{*}\right)^{1 /\left(1-\lambda_{k}\right)},\left(8 \Lambda b_{k}^{*}\right)^{1 /\left(1-\tau_{k}\right)}, k=1,2,3\right\}
$$

and define $U_{R}=\left\{(u, v) \in P:\|(u, v)\|_{X \times Y} \leq R\right\}$. For any $(u, v) \in U_{R}$, similar to (3.3) and (3.4), we obtain

$$
\sup _{t \in J} \frac{\left|\digamma_{1}(u, v)(t)\right|}{1+t^{\alpha+\beta-1}} \leq \Lambda\left[a_{0}^{*}+b_{0}^{*}+\sum_{k=1}^{3}\left(a_{k}^{*} R^{\lambda_{k}}+b_{k}^{*} R^{\tau_{k}}\right)\right] \leq R
$$


and

$$
\sup _{t \in J}\left|D^{\alpha-1} u(t)\right| \leq \Lambda\left[a_{0}^{*}+b_{0}^{*}+\sum_{k=1}^{3}\left(a_{k}^{*} R^{\lambda_{k}}+b_{k}^{*} R^{\tau_{k}}\right)\right] \leq R .
$$

This means that $\left\|\digamma_{1}(u, v)\right\|_{X} \leq R$. In the same way, $\left\|\digamma_{2}(u, v)\right\|_{Y} \leq R$ for all $(u, v) \in U_{R}$. Thus we know

$$
\|\digamma(u, v)\|_{X \times Y}=\left\{\left\|\digamma_{1}(u, v)\right\|_{X},\left\|\digamma_{2}(u, v)\right\|_{Y}\right\} \leq R .
$$

That is, $\digamma\left(U_{R}\right) \subset U_{R}$.

Through (3.8) and (3.9), it is obvious that $\left(u_{0}(t), v_{0}(t)\right),\left(w_{0}(t), z_{0}(t)\right) \in U_{R}$. By the complete continuity of the operator $\digamma$, we define the sequences $\left(u_{n}, v_{n}\right)$ and $\left(w_{n}, z_{n}\right)$ as $\left(u_{n}, v_{n}\right)=$ $\digamma\left(u_{n-1}, v_{n-1}\right),\left(w_{n}, z_{n}\right)=\digamma\left(w_{n-1}, z_{n-1}\right)$ for $n=1,2, \ldots$ Since $\digamma\left(U_{R}\right) \subset U_{R}$, we can obtain that $\left(u_{n}, v_{n}\right),\left(w_{n}, z_{n}\right) \in \digamma\left(U_{R}\right)$ for $n=1,2, \ldots$. Hence we need to prove that there exist $\left(u^{*}, v^{*}\right)$ and $\left(w^{*}, z^{*}\right)$ satisfying $\lim _{n \rightarrow \infty}\left(u_{n}, v_{n}\right)=\left(u^{*}, v^{*}\right)$ and $\lim _{n \rightarrow \infty}\left(w_{n}, z_{n}\right)=\left(w^{*}, z^{*}\right)$, which are two monotone sequences for positive solutions of the fractional differential system (1.6).

For $t \in J$, by Lemma 2.5, (3.1), and (3.8), we have

$$
u_{1}(t)=\digamma_{1}\left(u_{0}, v_{0}\right)(t) \leq t^{\alpha-1} \Lambda\left[a_{0}^{*}+\sum_{k=1}^{3} a_{k}^{*} R^{\lambda_{k}}+b_{0}^{*}+\sum_{k=1}^{3} b_{k}^{*} R^{\tau_{k}}\right] \leq R t^{\alpha-1}=u_{0}(t)
$$

and

$$
v_{1}(t)=\digamma_{2}\left(u_{0}, v_{0}\right)(t) \leq t^{\beta-1} \Lambda\left[b_{0}^{*}+\sum_{k=1}^{3} b_{k}^{*} R^{\tau_{k}}+a_{0}^{*}+\sum_{k=1}^{3} a_{k}^{*} R^{\lambda_{k}}\right] \leq R t^{\beta-1}=v_{0}(t)
$$

that is,

$$
\left(\begin{array}{l}
u_{1}(t) \\
v_{1}(t)
\end{array}\right)=\left(\begin{array}{l}
\digamma_{1}\left(u_{0}, v_{0}\right)(t) \\
\digamma_{2}\left(u_{0}, v_{0}\right)(t)
\end{array}\right) \leq\left(\begin{array}{l}
R t^{\alpha-1} \\
R t^{\beta-1}
\end{array}\right)=\left(\begin{array}{l}
u_{0}(t) \\
v_{0}(t)
\end{array}\right)
$$

Then we consider the monotonicity of the fractional derivative of $(u, v)$. By (3.12) and Remark 2.6, we know

$$
\begin{aligned}
D^{\alpha-1} u_{1}(t) & =D^{\alpha-1} \digamma_{1}\left(u_{0}, v_{0}\right)(t)=\int_{0}^{+\infty} H_{1}(t, s) \varphi_{\left(u_{0}, v_{0}\right)}(s) \mathrm{d} s+\int_{0}^{+\infty} H_{3}(t, s) \psi_{\left(u_{0}, v_{0}\right)}(s) \mathrm{d} s \\
& \leq \Gamma(\alpha) \Lambda\left[a_{0}^{*}+\sum_{k=1}^{3} a_{k}^{*} R^{\lambda_{k}}+b_{0}^{*}+\sum_{k=1}^{3} b_{k}^{*} R^{\tau_{k}}\right] \leq \Gamma(\alpha) R=D^{\alpha-1} u_{0}(t), \\
D^{\beta-1} v_{1}(t) & =D^{\beta-1} T_{2}\left(u_{0}, v_{0}\right)(t)=\int_{0}^{+\infty} H_{2}(t, s) \psi_{\left(u_{0}, v_{0}\right)}(s) \mathrm{d} s+\int_{0}^{+\infty} H_{4}(t, s) \varphi_{\left(u_{0}, v_{0}\right)}(s) \mathrm{d} s \\
& \leq \Gamma(\beta) \Lambda\left[a_{0}^{*}+\sum_{k=1}^{3} a_{k}^{*} R^{\lambda_{k}}+b_{0}^{*}+\sum_{k=1}^{3} b_{k}^{*} R^{\tau_{k}}\right] \leq \Gamma(\beta) R=D^{\beta-1} v_{0}(t),
\end{aligned}
$$

that is,

$$
\left(\begin{array}{l}
D^{\alpha-1} u_{1}(t) \\
D^{\beta-1} v_{1}(t)
\end{array}\right)=\left(\begin{array}{l}
D^{\alpha-1} \digamma_{1}\left(u_{0}, v_{0}\right)(t) \\
D^{\beta-1} \digamma_{2}\left(u_{0}, v_{0}\right)(t)
\end{array}\right) \leq\left(\begin{array}{c}
\Gamma(\alpha) R \\
\Gamma(\beta) R
\end{array}\right)=\left(\begin{array}{c}
D^{\alpha-1} u_{0}(t) \\
D^{\beta-1} v_{0}(t)
\end{array}\right)
$$


By the monotonicity assumption (C3) of functions $\varphi$ and $\psi$, similar to (3.12) and (3.13), for $\forall t \in J$, we do the second iteration:

$$
\begin{aligned}
& \left(\begin{array}{l}
u_{2}(t) \\
v_{2}(t)
\end{array}\right)=\left(\begin{array}{l}
\digamma_{1}\left(u_{1}, v_{1}\right)(t) \\
\digamma_{2}\left(u_{1}, v_{1}\right)(t)
\end{array}\right) \leq\left(\begin{array}{c}
\digamma_{1}\left(u_{0}, v_{0}\right)(t) \\
\digamma_{2}\left(u_{0}, v_{0}\right)(t)
\end{array}\right)=\left(\begin{array}{l}
u_{1}(t) \\
v_{1}(t)
\end{array}\right), \\
& \left(\begin{array}{l}
D^{\alpha-1} u_{2}(t) \\
D^{\beta-1} v_{2}(t)
\end{array}\right)=\left(\begin{array}{l}
D^{\alpha-1} \digamma_{1}\left(u_{1}, v_{1}\right)(t) \\
D^{\beta-1} \digamma_{2}\left(u_{1}, v_{1}\right)(t)
\end{array}\right) \leq\left(\begin{array}{c}
D^{\alpha-1} \digamma_{1}\left(u_{0}, v_{0}\right)(t) \\
D^{\beta-1} \digamma_{2}\left(u_{0}, v_{0}\right)(t)
\end{array}\right)=\left(\begin{array}{l}
D^{\alpha-1} u_{1}(t) \\
D^{\beta-1} v_{1}(t)
\end{array}\right) .
\end{aligned}
$$

By recursion, for $t \in J$, the sequence $\left\{\left(u_{n}, v_{n}\right)\right\}_{n=0}^{\infty}$ satisfies

$$
\left(\begin{array}{l}
u_{n+1}(t) \\
v_{n+1}(t)
\end{array}\right) \leq\left(\begin{array}{l}
u_{n}(t) \\
v_{n}(t)
\end{array}\right), \quad\left(\begin{array}{l}
D^{\alpha-1} u_{n+1}(t) \\
D^{\beta-1} v_{n+1}(t)
\end{array}\right) \leq\left(\begin{array}{l}
D^{\alpha-1} u_{n}(t) \\
D^{\beta-1} v_{n}(t)
\end{array}\right)
$$

Applying the iterative sequence $\left(u_{n+1}, v_{n+1}\right)=\digamma\left(u_{n}, v_{n}\right)$ and the complete continuity of the operator $\digamma$, it is easy to infer that $\left(u_{n}, v_{n}\right) \rightarrow\left(u^{*}, v^{*}\right)$ and $\digamma\left(u^{*}, v^{*}\right)=\left(u^{*}, v^{*}\right)$. Thus $\left(u^{*}, v^{*}\right)$ is a fixed point of $\digamma$.

For the sequence $\left\{\left(w_{n}, z_{n}\right)\right\}_{n=0}^{\infty}$, we take a similar discussion. For $t \in J$, we attain

$$
\begin{aligned}
\left(\begin{array}{l}
w_{1}(t) \\
z_{1}(t)
\end{array}\right) & =\left(\begin{array}{l}
\digamma_{1}\left(w_{0}, z_{0}\right)(t) \\
\digamma_{2}\left(w_{0}, z_{0}\right)(t)
\end{array}\right)=\left(\begin{array}{l}
\int_{0}^{+\infty} K_{1}(t, s) \varphi_{\left(w_{0}, z_{0}\right)}(s) \mathrm{d} s+\int_{0}^{+\infty} K_{3}(t, s) \psi_{\left(w_{0}, z_{0}\right)}(s) \mathrm{d} s \\
\int_{0}^{+\infty} K_{2}(t, s) \psi_{\left(w_{0}, z_{0}\right)}(s) \mathrm{d} s+\int_{0}^{+\infty} K_{4}(t, s) \varphi_{\left(w_{0}, z_{0}\right)}(s) \mathrm{d} s
\end{array}\right) \\
\geq & \left(\begin{array}{l}
0 \\
0
\end{array}\right)=\left(\begin{array}{c}
w_{0}(t) \\
z_{0}(t)
\end{array}\right), \\
\left(\begin{array}{c}
D^{\alpha-1} w_{1}(t) \\
D^{\beta-1} z_{1}(t)
\end{array}\right) & =\left(\begin{array}{l}
\int_{0}^{+\infty} H_{1}(t, s) \varphi_{\left(w_{0}, z_{0}\right)}(s) \mathrm{d} s+\int_{0}^{+\infty} H_{3}(t, s) \psi_{\left(w_{0}, z_{0}\right)}(s) \mathrm{d} s \\
\int_{0}^{+\infty} H_{2}(t, s) \psi_{\left(w_{0}, z_{0}\right)}(s) \mathrm{d} s+\int_{0}^{+\infty} H_{4}(t, s) \varphi_{\left(w_{0}, z_{0}\right)}(s) \mathrm{d} s
\end{array}\right) \\
\geq & \left(\begin{array}{l}
0 \\
0
\end{array}\right)=\left(\begin{array}{c}
D^{\alpha-1} w_{0}(t) \\
D^{\beta-1} z_{0}(t)
\end{array}\right) .
\end{aligned}
$$

Using the the monotonicity assumption (C3) of functions $\varphi$ and $\psi$, we can obtain

$$
\begin{aligned}
& \left(\begin{array}{l}
w_{2}(t) \\
z_{2}(t)
\end{array}\right)=\left(\begin{array}{l}
\digamma_{1}\left(w_{1}, z_{1}\right)(t) \\
\digamma_{2}\left(w_{1}, z_{1}\right)(t)
\end{array}\right) \geq\left(\begin{array}{c}
\digamma_{1}\left(w_{0}, z_{0}\right)(t) \\
\digamma_{2}\left(w_{0}, z_{0}\right)(t)
\end{array}\right)=\left(\begin{array}{c}
w_{1}(t) \\
z_{1}(t)
\end{array}\right), \\
& \left(\begin{array}{c}
D^{\alpha-1} w_{2}(t) \\
D^{\beta-1} z_{2}(t)
\end{array}\right)=\left(\begin{array}{l}
D^{\alpha-1} \digamma_{1}\left(w_{1}, z_{1}\right)(t) \\
D^{\beta-1} \digamma_{2}\left(w_{1}, z_{1}\right)(t)
\end{array}\right) \geq\left(\begin{array}{l}
D^{\alpha-1} \digamma_{1}\left(w_{0}, z_{0}\right)(t) \\
D^{\beta-1} \digamma_{2}\left(w_{0}, z_{0}\right)(t)
\end{array}\right)=\left(\begin{array}{c}
D^{\alpha-1} w_{1}(t) \\
D^{\beta-1} z_{1}(t)
\end{array}\right) .
\end{aligned}
$$

Analogously, for $n=0,1,2, \ldots$ and $t \in J$, we know

$$
\left(\begin{array}{c}
w_{n+1}(t) \\
z_{n+1}(t)
\end{array}\right) \geq\left(\begin{array}{c}
w_{n}(t) \\
z_{n}(t)
\end{array}\right), \quad\left(\begin{array}{c}
D^{\alpha-1} w_{n+1}(t) \\
D^{\beta-1} z_{n+1}(t)
\end{array}\right) \geq\left(\begin{array}{c}
D^{\alpha-1} w_{n}(t) \\
D^{\beta-1} z_{n}(t)
\end{array}\right)
$$

Applying the iterative sequence $\left(w_{n+1}, z_{n+1}\right)=\digamma\left(w_{n}, z_{n}\right)$ and the complete continuity of the operator $\digamma$, it is easy to acquire that $\left(w_{n}, z_{n}\right) \rightarrow\left(w^{*}, z^{*}\right)$ and $\digamma\left(w^{*}, z^{*}\right)=\left(w^{*}, z^{*}\right)$. Thus $\left(w^{*}, z^{*}\right)$ is also a fixed point of $\digamma$.

Finally we prove that $\left(u^{*}, v^{*}\right)$ and $\left(w^{*}, z^{*}\right)$ are the minimal and maximal positive solutions of system (1.6). Suppose that $(\xi(t), \eta(t))$ is any positive solution of system (1.6), then 
$\digamma(\xi(t), \eta(t))=(\xi(t), \eta(t))$ and

$$
\begin{aligned}
& \left(\begin{array}{l}
w_{0}(t) \\
z_{0}(t)
\end{array}\right)=\left(\begin{array}{l}
0 \\
0
\end{array}\right) \leq\left(\begin{array}{l}
\xi(t) \\
\eta(t)
\end{array}\right) \leq\left(\begin{array}{l}
R t^{\alpha-1} \\
R t^{\beta-1}
\end{array}\right)=\left(\begin{array}{l}
u_{0}(t) \\
v_{0}(t)
\end{array}\right), \\
& \left(\begin{array}{l}
D^{\alpha-1} w_{0}(t) \\
D^{\beta-1} z_{0}(t)
\end{array}\right) \leq\left(\begin{array}{l}
D^{\alpha-1} \xi(t) \\
D^{\beta-1} \eta(t)
\end{array}\right) \leq\left(\begin{array}{l}
D^{\alpha-1} u_{0}(t) \\
D^{\beta-1} v_{0}(t)
\end{array}\right) .
\end{aligned}
$$

Using the monotone property of the operator $\digamma$, we obtain that

$$
\begin{aligned}
& \left(\begin{array}{l}
w_{1}(t) \\
z_{1}(t)
\end{array}\right)=\left(\begin{array}{l}
\digamma_{1}\left(w_{0}, z_{0}\right)(t) \\
\digamma_{2}\left(w_{0}, z_{0}\right)(t)
\end{array}\right) \leq\left(\begin{array}{l}
\xi(t) \\
\eta(t)
\end{array}\right) \leq\left(\begin{array}{l}
\digamma_{1}\left(u_{0}, v_{0}\right)(t) \\
\digamma_{2}\left(u_{0}, v_{0}\right)(t)
\end{array}\right)=\left(\begin{array}{l}
u_{1}(t) \\
v_{1}(t)
\end{array}\right), \\
& \left(\begin{array}{l}
D^{\alpha-1} w_{1}(t) \\
D^{\beta-1} z_{1}(t)
\end{array}\right) \leq\left(\begin{array}{l}
D^{\alpha-1} \xi(t) \\
D^{\beta-1} \eta(t)
\end{array}\right) \leq\left(\begin{array}{l}
D^{\alpha-1} u_{1}(t) \\
D^{\beta-1} v_{1}(t)
\end{array}\right) .
\end{aligned}
$$

Repeating the above process, we have

$$
\begin{aligned}
& \left(\begin{array}{l}
w_{n}(t) \\
z_{n}(t)
\end{array}\right) \leq\left(\begin{array}{l}
\xi(t) \\
\eta(t)
\end{array}\right) \leq\left(\begin{array}{l}
u_{n}(t) \\
v_{n}(t)
\end{array}\right), \\
& \left(\begin{array}{l}
D^{\alpha-1} w_{n}(t) \\
D^{\beta-1} z_{n}(t)
\end{array}\right) \leq\left(\begin{array}{l}
D^{\alpha-1} \xi(t) \\
D^{\beta-1} \eta(t)
\end{array}\right) \leq\left(\begin{array}{l}
D^{\alpha-1} u_{n}(t) \\
D^{\beta-1} v_{n}(t)
\end{array}\right) .
\end{aligned}
$$

Combining $\lim _{n \rightarrow \infty}\left(w_{n}, z_{n}\right)=\left(w^{*}, z^{*}\right)$ and $\lim _{n \rightarrow \infty}\left(u_{n}, v_{n}\right)=\left(u^{*}, v^{*}\right)$, the results (3.10) and (3.11) come naturally.

Again $\varphi(t, 0,0,0) \neq 0$ and $\psi(t, 0,0,0) \neq 0$ for all $t \in J$, we know that $(0,0)$ is not a solution of system (1.6). By (3.10) and (3.11), it is obvious that $\left(w^{*}, z^{*}\right)$ and $\left(u^{*}, v^{*}\right)$ are the extreme positive solutions of system (1.6), which can be constructed by means of two monotone iterative sequences in (3.8) and (3.9). Thus the proof is completed.

Remark 3.4 When the parameters $\lambda_{k}, \tau_{k}(k=1,2,3)$ take different values, the same result can be obtained by using a similar method, so we omit the details.

Example 3.5 Consider the following fractional differential system on a half-line:

$$
\left\{\begin{array}{l}
-D^{2.5} u(t)=\frac{2}{(10+t)^{2}}+\frac{e^{-t}|u(t)|^{0.1}}{\left(1+t^{3.6}\right)^{0.1}}+\frac{e^{-2 t}|v(t)|^{0.3}}{\left(1+t^{3.6}\right)^{0.3}}+\frac{\mid D^{1.1} v(t) 0^{0.4}}{1+t^{2}}, \\
-D^{2.1} v(t)=\frac{1}{(20+t)^{3}}+\frac{e^{-3 t}|u(t)|^{0.2}}{\left(1+t^{2.6}\right)^{0.2}}+\frac{e^{-4 t} \mid v(t) 0^{0.4}}{\left(1+t^{3.6}\right)^{0.4}}+\frac{3 t^{\mid}\left|D^{1.5} u(t)\right|^{0.2}}{\left(3+t^{3}\right)^{2}}, \\
u(0)=u^{\prime}(0)=0, \quad D^{1.5} u(+\infty)=\int_{0}^{+\infty} t^{-1.1} e^{-t} v(t) d t, \\
v(0)=v^{\prime}(0)=0, \quad D^{1.1} v(+\infty)=\int_{0}^{+\infty} t^{-1.5} e^{-2 t} u(t) d t,
\end{array}\right.
$$

where $\alpha=2.5, \beta=2.1, h(t)=t^{-1.1} e^{-t}, g(t)=t^{-1.5} e^{-2 t}, \lambda_{1}=0.1, \lambda_{2}=0.3, \lambda_{3}=0.4, \tau_{1}=0.2$, $\tau_{2}=0.4, \tau_{3}=0.2, \Gamma(2.5)=1.329340, \Gamma(2.1)=1.046486, \Upsilon_{1}=\int_{0}^{+\infty} h(t) t^{1.1} d t=1, \Upsilon_{2}=$ $\int_{0}^{+\infty} g(t) t^{1.5} d t=0.5$, and

$$
\varphi(t, u, v, w)=\frac{2}{(10+t)^{2}}+\frac{e^{-t}|u|^{0.1}}{\left(1+t^{3.6}\right)^{0.1}}+\frac{e^{-2 t}|v|^{0.3}}{\left(1+t^{3.6}\right)^{0.3}}+\frac{|w|^{0.4}}{1+t^{2}}
$$




$$
\psi(t, u, v, z)=\frac{1}{(20+t)^{3}}+\frac{e^{-3 t}|u|^{0.2}}{\left(1+t^{3.6}\right)^{0.2}}+\frac{e^{-4 t}|v|^{0.4}}{\left(1+t^{3.6}\right)^{0.4}}+\frac{3 t^{2}|z|^{0.2}}{\left(3+t^{3}\right)^{2}}
$$

It is easy to know that $\Gamma(2.5) \Gamma(2.1)>\Upsilon_{1} \Upsilon_{2}$. So assumptions $(\mathrm{C} 0)$ and $(\mathrm{C} 1)$ are satisfied.

Noting that

$$
\begin{aligned}
|\varphi(t, u, v, w)| & \leq \frac{2}{(10+t)^{2}}+\frac{e^{-t}|u|^{0.1}}{\left(1+t^{3.6}\right)^{0.1}}+\frac{e^{-2 t}|v|^{0.3}}{\left(1+t^{3.6}\right)^{0.3}}+\frac{|w|^{0.4}}{1+t^{2}} \\
& =a_{0}(t)+a_{1}(t)|u|^{0.1}+a_{2}(t)|v|^{0.3}+a_{3}(t)|w|^{0.4} \\
|\psi(t, u, v, z)| & \leq \frac{1}{(20+t)^{3}}+\frac{e^{-3 t}|u|^{0.2}}{\left(1+t^{3.6}\right)^{0.2}}+\frac{e^{-4 t}|v|^{0.4}}{\left(1+t^{3.6}\right)^{0.4}}+\frac{3 t^{2}|z|^{0.2}}{\left(3+t^{3}\right)^{2}} \\
& =b_{0}(t)+b_{1}(t)|u|^{0.2}+b_{2}(t)|v|^{0.4}+b_{3}(t)|z|^{0.2},
\end{aligned}
$$

and

$$
\begin{aligned}
& a_{0}^{*}=\int_{0}^{+\infty} a_{0}(t) d t=\frac{1}{5}, \quad a_{1}^{*}=\int_{0}^{+\infty} a_{1}(t)\left(1+t^{3.6}\right)^{0.1} d t=1, \\
& a_{2}^{*}=\int_{0}^{+\infty} a_{2}(t)\left(1+t^{3.6}\right)^{0.3} d t=\frac{1}{2}, \quad a_{3}^{*}=\int_{0}^{+\infty} a_{3}(t) d t=\frac{\pi}{2}, \\
& b_{0}^{*}=\int_{0}^{+\infty} b_{0}(t) d t=\frac{1}{800}, \quad b_{1}^{*}=\int_{0}^{+\infty} b_{1}(t)\left(1+t^{3.6}\right)^{0.2} d t=\frac{1}{3}, \\
& b_{2}^{*}=\int_{0}^{+\infty} b_{2}(t)\left(1+t^{3.6}\right)^{0.4} d t=\frac{1}{4}, \quad b_{3}^{*}=\int_{0}^{+\infty} b_{3}(t) d t=\pi,
\end{aligned}
$$

which means that assumption (C2) is satisfied.

From the expression of functions $\varphi, \psi$, it is obvious that $\varphi, \psi$ are increasing with respect to the variables $u, v, w$ and $u, v, z$, and $\varphi(t, 0,0,0) \not \equiv 0, \psi(t, 0,0,0) \not \equiv 0, \forall t \in J$. Thus assumption (C3) is satisfied. By Theorem 3.3, it follows that the fractional differential system (3.14) has two positive solutions, which can be established by the limit means of two explicit monotone iterative sequences in (3.8) and (3.9).

\section{Conclusions}

In this paper, we apply the monotone iterative technique to study a fractional differential system with coupled integral boundary conditions in a half-line. We first transform system (1.6) into an equivalent operator equation (3.1), and then we construct some norm inequalities related to nonlinear terms $\varphi, \psi$ and a new Banach space. Finally, some explicit monotone iterative sequences for approximating the extreme positive solutions are obtained.

\section{Acknowledgements}

Not applicable.

Funding

This work is supported by University Natural Science Foundation of Anhui Provincial Education Department (Grant No. KJ2017A442, KJ2018A0452), the Foundation of Suzhou University (Grant No. 2016XJGG13, 2019XJZY02), Technology Research Foundation of Chongqing Educational Committee(Grant No. KJQN201800533). 


\section{Competing interests}

The authors declare that they have no competing interests.

\section{Authors' contributions}

Each of the authors contributed to each part of this study equally, all authors read and approved the final manuscript.

\section{Author details}

${ }^{1}$ School of Mathematics and Statistics, Suzhou University, Suzhou 234000, Anhui, P.R. China. ${ }^{2}$ School of Mathematical Sciences, Qufu Normal University, Qufu 273165, Shandong, P.R. China. ${ }^{3}$ School of Mathematical Sciences, Chongqing Normal University, 401331 Chongqing, P.R. China.

\section{Publisher's Note}

Springer Nature remains neutral with regard to jurisdictional claims in published maps and institutional affiliations.

Received: 20 May 2020 Accepted: 27 July 2020 Published online: 03 August 2020

\section{References}

1. Kilbas, A.A., Srivastava, H.M., Trujillo, J.J.: Theory and Applications of Fractional Differential Equations. North-Holland Mathematics Studies, vol. 204. Elsevier, Amsterdam (2006)

2. Ahmad, B., Alsaed, A., Ntouyas, S.K., Tariboon, J.: Hadamard-Type Fractional Differential Equations, Inclusions and Inequalities. Springer, Berlin (2017)

3. Hao, X., Wang, H., Liu, L., Cui, Y.: Positive solutions for a system of nonlinear fractional nonlocal boundary value problems with parameters and p-Laplacian operator. Bound. Value Probl. 2017, 182 (2017)

4. Wang, Y., Liu, L.: Positive solutions for a class of fractional infinite-point boundary value problems. Bound. Value Probl. 2018, $118(2018)$

5. Wang, Y.: The Green's function of a class of two-term fractional differential equation boundary value problem and its applications. Adv. Differ. Equ. 2020, 80 (2020)

6. Wang, Y., Wang, H.: Existence and nonexistence of positive solutions for high-order fractional differential equation boundary value problems at resonance. J. Funct. Spaces 2020, 2395238 (2020)

7. Wang, Y., Wang, H.: Triple positive solutions for fractional differential equation boundary value problems at resonance. Appl. Math. Lett. 2020, 106376 (2020)

8. Zhang, X., Zhong, Q.: Triple positive solutions for nonlocal fractional differential equations with singularities both on time and space variables. Appl. Math. Lett. 80, 12-19 (2018)

9. Jiang, J., O'Regan, D., Xu, J., Fu, Z.: Positive solutions for a system of nonlinear Hadamard fractional differential equations involving coupled integral boundary conditions. J. Inequal. Appl. 2019, 204 (2019)

10. Zhang, H., Li, Y., Xu, J.: Positive solutions for a system of fractional integral boundary value problems involving Hadamard-type fractional derivatives. Complexity 2019, 2671539 (2019)

11. Zhao, Y., Xu, J., Chen, H.: Variational methods for an impulsive fractional differential equations with derivative term. Mathematics 7, 880 (2019)

12. Cheng, W., Xu, J., O'Regan, D., Cui, Y.: Positive solutions for a nonlinear discrete fractional boundary value problem with a p-Laplacian operator. J. Appl. Anal. Comput. 9, 1959-1972 (2019)

13. $\mathrm{Xu}$, J., Jiang, J., O'Regan, D.: Positive solutions for positive solutions for a class of $p$-Laplacian Hadamard fractional-order three-point boundary value problems. Mathematics 8, 308 (2020)

14. Ding, Y., Jiang, J., O'Regan, D., Xu, J.: Positive solutions for a system of Hadamard-type fractional differential equations with semipositone nonlinearities. Complexity 2020, 9742418 (2020)

15. Zhang, H., Li, Y., Yang, J.: New sequential fractional differential equations with mixed-type boundary conditions. J. Funct. Spaces 2020, 6821637 (2020)

16. Wang, G., Baleanu, D., Zhang, L.: Monotone iterative method for a class of nonlinear fractional differential equations. Fract. Calc. Appl. Anal. 15, 244-252 (2012)

17. Cui, Y., Sun, Q., Su, X.: Monotone iterative technique for nonlinear boundary value problems of fractional order $p \in(2,3]$. Adv. Differ. Equ. 2017, 1 (2017)

18. Zhang, X., Wu, J., Liu, L., Wu, Y., Cui, Y.: Convergence analysis of iterative scheme and error estimation of positive solution for a fractional differential equation. Math. Model. Anal. 23, 611-626 (2018)

19. Guo, L., Liu, L., Wu, Y.: Iterative unique positive solutions for singular p-Laplacian fractional differential equation system with several parameters. Nonlinear Anal., Model. Control 23, 182-203 (2018)

20. Wei, Y., Song, Q., Bai, Z.: Existence and iterative method for some fourth order nonlinear boundary value problems. Appl. Math. Lett. 87, 101-107 (2019)

21. Guo, L., Liu, L.: Unique iterative positive solutions for a singular $p$-Laplacian fractional differential equation system with infinite-point boundary conditions. Bound. Value Probl. 2019, 113 (2019)

22. Yang, W.: Monotone iterative technique for a coupled system of nonlinear Hadamard fractional differential equations. J. Appl. Math. Comput. 59, 585-596 (2019)

23. Wang, F., Liu, L., Wu, Y., Zou, Y.: Iterative analysis of the unique positive solution for a class of singular nonlinear boundary value problems involving two types of fractional derivatives with $p$-Laplacian operator. Complexity 2019 2319062 (2019)

24. Wang, G., Qin, J., Zhang, L., Baleanu, D.: Explicit iteration to a nonlinear fractional Langevin equation with non-separated integro-differential strip-multi-point boundary conditions. Chaos Solitons Fractals 131, 109476 (2020)

25. Zhang, S.: Monotone iterative method for initial value problem involving Riemann-Liouville fractional derivatives. Nonlinear Anal., Theory Methods Appl. 71, 2087-2093 (2009)

26. Zhang, L., Ahmad, B., Wang, G.: Successive iterations for positive extremal solutions of nonlinear fractional differential equations on a half-line. Bull. Aust. Math. Soc. 91, 116-128 (2015) 
27. Pei, K., Wang, G., Sun, Y.: Successive iterations and positive extremal solutions for a Hadamard type fractional integro-differential equations on infinite domain. Appl. Math. Comput. 312, 158-168 (2017)

28. Zhai, C., Wei, L.: The unique positive solution for fractional integro-differential equations on infinite intervals. ScienceAsia 312, 118-124 (2018)

29. Zhai, C., Ren, J.: A coupled system of fractional differential equations on the half-line. Bound. Value Probl. 2019, 117 (2019)

30. Ntouyas, S.K., Tariboon, J., Thaiprayoon, C.: Nonlocal boundary value problems for Riemann-Liouville fractional differential inclusions with Hadamard fractional integral boundary conditions. Taiwan. J. Math. 20, 91-107 (2016)

31. Fu, Z., Bai, S., O'Regan, D., Xu, J.: Nontrivial solutions for an integral boundary value problem involving Riemann-Liouville fractional derivatives. J. Inequal. Appl. 2019, 104 (2019)

32. Aljoudi, S., Ahmad, B., Nieto, J.J., Ahmed, A.: A coupled system of Hadamard type sequential fractional differential equations with coupled strip conditions. Chaos Solitons Fractals 91, 39-46 (2016)

33. Li, Y., Sang, Y., Zhang, H.: Solvability of a coupled system of nonlinear fractional differential equations with fractional integral conditions. J. Appl. Math. Comput. 50, 73-91 (2016)

34. Liu, B., Li, J., Liu, L., Wang, Y.: Existence and uniqueness of nontrivial solutions to a system of fractional differential equations with Riemann-Stieltjes integral conditions. Adv. Differ. Equ. 2018, 306 (2018)

35. Riaz, U., Zada, A., Ali, Z., Cui, Y., Xu, J.: Analysis of coupled systems of implicit impulsive fractional differential equations involving Hadamard derivatives. Adv. Differ. Equ. 2019, 226 (2019)

36. Zhang, X., Liu, L., Wu, Y., Zou, Y.: Existence and uniqueness of solutions for systems of fractional differential equations with Riemann-Stieltjes integral boundary condition. Adv. Differ. Equ. 2018, 204 (2018)

37. Xu, J., Goodrich, C.S., Cui, Y.: Positive solutions for a system of first-order discrete fractional boundary value problems with semipositone nonlinearities. Rev. R. Acad. Cienc. Exactas Fís. Nat., Ser. A Mat. 113, 1343-1358 (2019)

38. Cheng, W., Xu, J., Cui, Y., Ge, Q.: Positive solutions for a class of fractional difference systems with coupled boundary conditions. Adv. Differ. Equ. 2019, 249 (2019)

39. Wang, F., Cui, Y.: Positive solutions for an infinite system of fractional order boundary value problems. Adv. Differ. Equ. 2019, $169(2019)$

40. Ding, Y., Xu, J., Fu, Z.: Positive solutions for a system of fractional integral boundary value problems of Riemann-Liouville type involving semipositone nonlinearities. Mathematics 7, 970 (2019)

41. Zhao, D., Liu, Y.: Twin solutions to semipositone boundary value problems for fractional differential equations with coupled integral boundary conditions. J. Nonlinear Sci. Appl. 10, 3544-3565 (2017)

42. Wang, Y., Liu, Y., Cui, Y.: Multiple sign-changing solutions for nonlinear fractional Kirchhoff equations. Bound. Value Probl. 2018, 193 (2018)

43. Wang, Y., Liu, Y., Cui, Y.: Infinitely many solutions for impulsive fractional boundary value problem with $p$-Laplacian. Bound. Value Probl. 2018, 94 (2018)

44. Su, X., Zhang, S.: Unbounded solutions to a boundary value problem of fractional order on the half-line. Comput. Math. Appl. 61, 1079-1087 (2011)

\section{Submit your manuscript to a SpringerOpen ${ }^{\mathcal{O}}$ journal and benefit from:}

- Convenient online submission

- Rigorous peer review

- Open access: articles freely available online

- High visibility within the field

- Retaining the copyright to your article

Submit your next manuscript at $\gg$ springeropen.com 